\title{
A PHY Layer Security Analysis of Uplink Cooperative Jamming-Based Underlay CRNs with Multi-Eavesdroppers
}

\author{
Mounia Bouabdellah, Faissal El Bouanani, Senior \\ Member, IEEE, and Mohamed-Slim Alouini, Fellow, IEEE
}

\begin{abstract}
In this paper, the physical layer security of a dual-hop underlay uplink cognitive radio network is investigated over Nakagami- $m$ fading channels. Specifically, multiple secondary sources $\left(S_{i}\right)_{1 \leq i \leq N}$ are taking turns in accessing the licensed spectrum of the primary users and communicating with a multi-antenna secondary base station $(D)$ through the aid of a multi-antenna relay $R$ in the presence of $M$ eavesdroppers $\left(E_{k}\right)_{1 \leq k \leq M}$ that are also equipped with multiple antennas. Among the remaining nodes, one jammer is randomly selected to transmit an artificial noise to disrupt all the eavesdroppers that are attempting to intercept the communication of the legitimate links i.e., $S_{i}-R$ and $R-D$. The received signals at each node are combined using maximum-ratio combining. Secrecy analysis is provided by deriving closed-form and asymptotic expressions for the secrecy outage probability. The impact of several key parameters on the system's secrecy e.g., transmit power of the sources, number of eavesdroppers, maximum tolerated interference power, and the number of diversity branches is investigated. Importantly, by considering two scenarios, namely (i) absence and (ii) presence of a friendly jammer, new insights are obtained for the considered communication system. Especially, we tend to answer to the following question: Can better secrecy be achieved without jamming by considering a single antenna at eavesdroppers and multiple-ones at the legitimate users (i.e., relay and end-user) rather than sending permanently an artificial noise and considering that both the relay and the destination are equipped with a single antenna, while multiple antennas are used by the eavesdroppers? The obtained
\end{abstract}

M. Bouabdellah and F. El Bouanani are with ENSIAS College of Engineering, Mohammed V University, Rabat, Morocco (e-mails: mounia_bouabdellah@um5.ac.ma,f.elbouanani@um5s.net.ma).

M.-S. Alouini is with Computer, Electrical, and Mathematical Sciences and Engineering (CEMSE) Division, King Abdullah University of Science and Technology (KAUST), Thuwal 23955-6900, Makkah Province, Saudi Arabia (e-mail: slim.alouini@kaust.edu.sa). 
results are corroborated through Monte Carlo simulation and show that the system's security can be enhanced by adjusting the aforementioned parameters.

\section{Index Terms}

Cognitive Radio Networks, Eavesdropping, Jamming signals, Physical Layer Security, Secrecy outage probability.

\section{INTRODUCTION}

The increasing number of mobile users led to an unprecedented demand for spectral resources. In this regard, cognitive radio has emerged as a new paradigm that enhances the spectrum efficiency by allowing its reuse [1]. In underlay cognitive radio networks (CRNs), the issue of radio-frequency spectrum scarcity is alleviated by allowing the secondary users (SUs) to share the spectrum with primary users (PUs) under the condition of not causing any harmful interference to them. Consequently, the SUs are required to continuously adjust their transmit powers in order to meet the PUs' quality of service (QoS). Under such constraints, ensuring the physical layer security (PLS) of multi-hop CRNs becomes a challenge of utmost importance. To remedy this problem, several techniques can be used to strengthen the secrecy capacity at each hop namely increasing the number of diversity branches at the receivers, sending a jamming signal with the highest power, increasing maximum transmit power at the source and maximum tolerated interference power as well, reducing the number of hops, employing zeroforcing precoding techniques, involving energy harvesting $(\mathrm{EH})$ and non-orthogonal multiple access (NOMA) technique etc.

Recently, the PLS of CRNs has been the focus of many recent research works. For instance, non-cooperative CRNs were considered in [2]-[5], therein all receivers i.e., both destination and eavesdropper were assumed to be equipped with multiple antennas and perform the selection combining (SC) technique. Particularly, in [2], the source is also assumed to be a multi-antennas node performing transmit antenna selection, while in [3] the secrecy performance is investigated for both secondary and primary networks. Closed-form and asymptotic expressions for the secrecy outage probability (SOP) were derived under Rayleigh [3], [4] and Nakagami-m [2], [5] fading models.

The PLS of multi-relays dual-hop CRNs was explored in [6]-[9]. Specifically, in [6] and [7], the communication was performed in the presence of only one eavesdropper attempting to 
overhear the communication channel, while multiple eavesdroppers were considered in [8] and [9]. Furthermore, In [6], optimal and suboptimal relay selection were analyzed while in [7] the relay that minimizes the signal-to-noise ratio (SNR) of the wiretap link was chosen. Besides, in [8], the most threatening eavesdropper is selected first according to the maximum SNR of the wiretap links between the source and the eavesdroppers. Next, the best relay minimizing the SNR at the selected eavesdropper is then chosen. In [9], the relay that maximizes the achievable secrecy rate is selected. Under these conditions, closed-form and asymptotic expressions for the SOP and intercept probability (IP) were derived over either Nakagami-m [6] or Rayleigh [7]-[9] fading channels. The IP and SOP analysis of cooperative underlay EH-based CRNs have been investigated in [10] and [10]-[14], respectively. Specifically, the SUs have been assumed to harvest energy from the PU's signals in [10]-[12]. In contrast, in [13]-[14] the relay is harvesting energy from the SU signals instead.

The PLS of NOMA-based CRNs has been investigated in [15]-[16]. In [15], an overlay NOMA CRN was considered such that the SUs were assumed to be eavesdroppers, while the PLS of mmWave NOMA CRN was investigated in [16]. Closed-form expressions for the connection outage probability, SOP and secrecy throughput were derived over Nakagami- $m$ fading channels.

PLS analysis through the aid of a friendly jammer was discussed in [17]-[19]. In [17], the IP was derived by considering multiple source-destination pairs communicating under eavesdropping attempts of only one eavesdropper, with the source cooperation aided opportunistic jamming. In [18], the SOP of dual-hop aided opportunistic jamming CRNs is investigated. In this work, one relay is selected to forward the information while another one is chosen to disrupt the eavesdropper by sending an artificial noise. Also, in the two aforementioned works, several selection policies of the friendly jammer were considered. The impact of the friendly jammer's transmit power in the presence of multiple eavesdroppers by considering a direct communication link between multiple sources and one destination is discussed in [19].

In this work, we investigate the joint impact of the friendly jammer's transmit power, multiple SUs with power adaptation constraint, number of eavesdroppers, number of diversity branches, maximum tolerated interference power at the PU receiver on the PLS of a cooperative underlay uplink CRNs under Nakagami- $m$ fading model. Without loss of generality, it is worth mentioning that each user is transmitting its data independently from other users. Consequently, the sources are assumed to transmit in turns their data while a friendly jammer is randomly selected among the remaining idle sources to transmit an artificial noise so as to disrupt the eavesdroppers. In 
this scheme, the nodes $R, D, E_{k}$ perform MRC technique, hence the knowledge of the channel state information (CSI) at these nodes is necessary. For this reason, we assume that the CSI is available. Additionally, eavesdroppers are considered are passive.

The main contributions of this paper can be summarized as follows:

- The PLS of an underlay uplink dual-hop CRN operating under Nakagami- $m$ fading environment is investigated by deriving closed-form and asymptotic expressions for the SOP of the overall system under two scenarios namely, (i) presence and (ii) absence of a friendly jammer.

- Under the power adaptation constraint of the SUs, the joint impact of the discussed parameters on the system's security is investigated.

- We show that the system's security is enhanced in the presence of an important number of eavesdroppers by increasing the (i) SUs' transmit powers (ii) number of legitimate destination branches (iii) and maximum tolerated interference power.

The rest of this paper is organized as follows. In Section II, the system and channel models are presented. Closed-form as well as asymptotic expressions for the SOP are derived in Section III. In Section IV, the numerical and simulation results are provided and discussed for various key parameters' values. Finally, this work is concluded in Section V.

\section{SYSTEM AND CHANNEL MODELS}

The considered two-hops CRN, represented in Fig. 1, consists of multiple sources $\left(S_{i}\right)_{i=1, . ., N}$, one $L_{R}$-antennas relay $R$, multiple $L_{E_{k}}$-antennas eavesdroppers $\left(E_{k}\right)_{k=1, . ., M}$, one destination $D$ equipped with $L_{D}$ antennas, one PU transmitter $\left(P_{T x}\right)$, and one PU receiver $\left(P_{R x}\right)$. For the sake of simplicity, we assume that the relay receives the transmitted signals from $S_{i}$ on the $L_{R}$ antennas and uses only one antenna to forward the message to $D$. Moreover, we consider multiuser scheduling such that, at any given moment, only one user is transmitting its data. Also, the source nodes are taking rounds in accessing the spectrum and a friendly jammer $S_{J}$ is randomly selected among $N-1$ remaining nodes to send an artificial noise. This latter can be canceled by legitimate nodes, while $E_{k}$ cannot mitigate it, leading to an increase in the secrecy capacity. Similarly to [17], we assume that a friendly jammer generates an artificial noise using a pseudorandom sequence that is known to the legitimate users which allows them to cancel out this noise, while this sequence remains unknown to the illegitimate ones. To this end, the main aim of this work is to investigate the impact of a friendly jammer, legitimate, and wiretap channels' 
average SNRs, maximum tolerated interference power as well as the spatial diversity at both the relay and the end-user on the secrecy performance of the considered communication system. In this scheme, Nakagami- $m$ fading model is considered for all links. The fading amplitudes of links $S_{i} \rightarrow R, R \rightarrow\left(D_{t}\right)_{1 \leq t \leq L_{D}}, S_{i} \rightarrow E_{k}, R \rightarrow E_{k}, R \rightarrow P_{R x}, S_{i} \rightarrow P_{R x}$ are denoted by $h_{q}$ where $q=\left\{S_{i} R, R D_{t}, S_{i} E_{k}, R E_{k}, R P, S_{i} P\right\}$. Consequently, the channel gains $g_{q}=\left|h_{q}\right|^{2}$ are Gamma distributed with probability density function (PDF) and cumulative density function (CDF) are given by

$$
\begin{gathered}
f_{g_{q}}(x)=\frac{\lambda_{q}^{m_{q}}}{\Gamma\left(m_{q}\right)} x^{m_{q}-1} e^{-\lambda_{q} x}, \\
F_{g_{q}}(x)=\frac{\gamma\left(m_{q}, \lambda_{q} x\right)}{\Gamma\left(m_{q}\right)},
\end{gathered}
$$

where $\lambda_{q}=\frac{m_{q}}{\Omega_{q}}, m_{q}$ and $\Omega_{q}$ denote the fading severity and the average channel power gain, respectively, $\Gamma($.$) and \gamma(.,$.$) are the Euler and the lower incomplete Gamma functions [21, Eqs.$ (8.310.1), (8.350.1)], respectively. For a natural number $m_{q}$, the above CDF can be written as [13, Eq. (8.352.1)]

$$
F_{g_{q}}(x)=1-e^{-\lambda_{q} x} \sum_{k=0}^{m_{q}-1} \frac{\lambda_{q}^{k} x^{k}}{k !} .
$$

The received signals at $R, E_{k}$ at both hops, and $D$ are given, respectively, by

$$
\begin{gathered}
y_{R}^{(i)}=\sqrt{P_{S_{i}}}\left\|\mathbf{h}_{S_{i} R}\right\| x_{S_{i}}+\mathbf{w}_{S_{i} R} \mathbf{n}_{R}, i=1, . ., N, \\
y_{1 E_{k}}^{(i)}=\sqrt{P_{S_{i}}}\left\|\mathbf{h}_{S_{i} E_{k}}\right\| x_{S_{i}}+\epsilon \sqrt{P_{S_{J}}}|| \mathbf{h}_{S_{J} E_{k}} \| x_{S_{J}}+\mathbf{w}_{S_{i} E_{k}} \mathbf{n}_{E_{k}}, \\
k=1, . ., M, i=1, . ., N, J \neq i \\
y_{2 E_{k}}=\sqrt{P_{R}}\left\|\mathbf{h}_{R E_{k}}\right\| x_{R}+\mathbf{w}_{R E_{k}} \mathbf{n}_{E_{k}}, k=1, . ., M, \\
y_{D}=\sqrt{P_{R}}|| \mathbf{h}_{R D} \| x_{R}+\mathbf{w}_{R D} \mathbf{n}_{D},
\end{gathered}
$$

with

$$
\epsilon=\left\{\begin{array}{l}
0, \text { Absence of a jammer } \\
1, \text { Presence of a jammer }
\end{array} .\right.
$$

Here, $P_{n}$ and $x_{n}$ denote the transmit power and signal from the node $n$, respectively where $n=\left\{S_{i}, R\right\}, \mathbf{w}_{q}=\frac{\mathbf{h}_{q}^{\dagger}}{\left\|\mathbf{h}_{q}\right\|}, q=\left\{S_{i} R, S_{i} E_{k}, R E_{k}, R D\right\}$, while $\mathbf{h}_{q}$ denotes $L_{n} \times 1$, channel vector of the links $S_{i}-R, S_{i}-E_{k}, R-D$, $\dagger$ denotes the transpose conjugate, and \|.|| represents the Frobenius norm. Also, $\mathbf{n}_{R}, \mathbf{n}_{D}$, and $\mathbf{n}_{E_{k}}$, denote the $N_{n} \times 1$ additive white Gaussian noise vector 
at $R, D$, and $E_{k}$, respectively. For the sake of simplicity, all noise power vectors' components are considered equal $N_{0}$.

Throughout the transmission process, both $S_{i}$, and $R$ have to adapt their transmit powers so as to avoid causing harmful interference to the PUs. Thus, the transmit power of the source and the relay $R$ taking into consideration the maximum constraint power can be, respectively, expressed as

$$
P_{S_{i}}=\min \left(P_{S_{i}}^{\max }, \frac{P_{I}}{g_{S_{i} P}}\right) ; i=1, . ., N
$$

and

$$
P_{R}=\min \left(P_{R}^{\max }, \frac{P_{I}}{g_{R P}}\right),
$$

where $P_{S_{i}}^{\max }$ and $P_{R}^{\max }$ denote the maximum transmit power at $S_{i}$, and $R$, respectively, while $P_{I}$ accounts for the maximum tolerated interference power at $P_{R x}$.

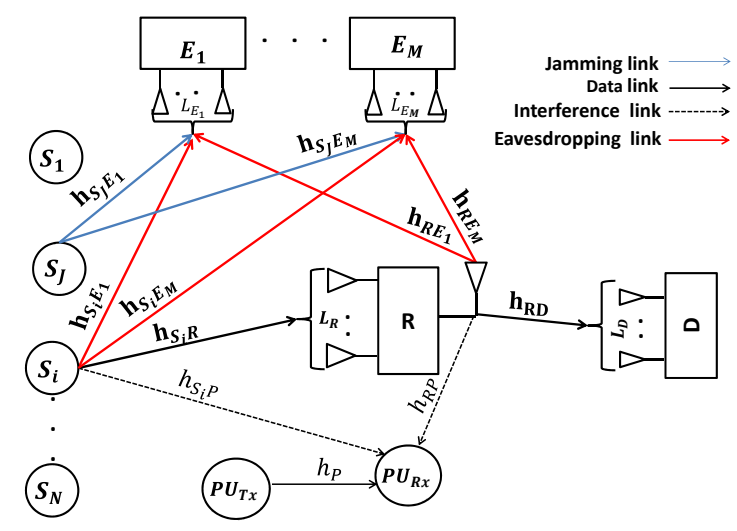

Fig. 1: System setup.

\section{SECRECY OUTAGE PROBABILITY}

In this section, we start by defining the secrecy capacities for the two hops' links. Next, we present the SOP analysis in terms of both closed-form and asymptotic expressions for various cases of fading severity parameters, specifically, those of the legitimate and the wiretap links of the first hop. Also, two scenarios will be considered, namely, the presence and absence of a friendly jammer. 


\section{A. Secrecy Capacity}

The secrecy capacity can be defined as the maximum rate at which the transmitted information can confidentially reach its intended destination. In our considered system, the secrecy capacities in the case of presence and absence of a friendly jammer are given, respectively, by

$$
\begin{aligned}
C_{s}^{(i, J)} & =\min _{k=1, . ., M}\left(C_{1 S}^{(i, k, J)}, C_{2 S}^{(k)}\right), \\
C_{s}^{(i)} & =\min _{k=1, . ., M}\left(C_{1 S}^{(i, k)}, C_{2 S}^{(k)}\right),
\end{aligned}
$$

where

- $C_{1 S}^{(i, k, J)}$ and $C_{1 S}^{(i, k)}$ denote the secrecy capacities at the first hop, i.e., the difference between the capacity of the main link $S_{i}-R$ and the one of the wiretap channel $S_{i}-E_{k}$ in the presence and absence of a friendly jammer, respectively, and can be written as

$$
\begin{aligned}
& C_{1 S}^{(i, k, J)}=\left\{\begin{array}{l}
\log _{2}\left(\frac{1+\gamma_{R}^{(i)}}{1+\gamma_{1 E}^{(i, k, J)}}\right), \gamma_{R}^{(i)}>\gamma_{1 E}^{(i, k, J)} \\
0, \text { elsewhere }
\end{array}\right. \\
& C_{1 S}^{(i, k)}=\left\{\begin{array}{l}
\log _{2}\left(\frac{1+\gamma_{R}^{(i)}}{1+\gamma_{1 E}^{(i, k)}}\right), \gamma_{R}^{(i)}>\gamma_{1 E}^{(i, k)} \\
0, \text { elsewhere }
\end{array}\right.
\end{aligned}
$$

where $\gamma_{R}^{(i)}$ denotes the instantaneous SNR at $R$, while $\gamma_{1 E}^{(i, k, J)}$ and $\gamma_{1 E}^{(i, k)}$ stand for the SNRs at the eavesdropper $E_{k}$ in the presence and absence of a friendly jammer, respectively, and are given by

$$
\begin{gathered}
\gamma_{R}^{(i)}=\min \left(\bar{\gamma}_{S_{i}}, \frac{\bar{\gamma}_{I}}{g_{S_{i} P}}\right) \sum_{u=1}^{L_{R}} g_{S_{i} R_{u}}, \\
\gamma_{1 E}^{(i, k, J)}=\frac{\min \left(\bar{\gamma}_{S_{i}}, \frac{\bar{\gamma}_{I}}{g_{S_{i} P}}\right) \sum_{u=1}^{L_{E_{k}}} g_{S_{i} E_{k}^{(u)}}}{\min \left(\bar{\gamma}_{S_{J}}, \frac{\bar{\gamma}_{I}}{g_{S_{J} P}}\right) \sum_{u=1}^{L_{E_{k}}} g_{S_{J} E_{k}^{(u)}}+1}, \\
\gamma_{1 E}^{(i, k)}=\min \left(\bar{\gamma}_{S_{i}}, \frac{\bar{\gamma}_{I}}{g_{S_{i} P}}\right) \sum_{u=1}^{L_{E_{k}}} g_{S_{i} E_{k}^{(u)}},
\end{gathered}
$$

and $\bar{\gamma}_{S_{i}}=P_{S_{i}}^{\max } / N_{0}, \bar{\gamma}_{I}=P_{I} / N_{0}$, and $\bar{\gamma}_{S_{J}}=P_{S_{J}}^{\max } / N_{0}$ 
- $C_{2 S}^{(k)}$ is the secrecy capacity of the second hop, representing the difference between the capacity of the link $R-D$ and the one of the wiretap channel $R-E_{k}$

$$
C_{2 S}^{(k)}=\left\{\begin{array}{l}
\log _{2}\left(\frac{1+\gamma_{D}}{1+\gamma_{2 E}^{(k)}}\right), \gamma_{D}>\gamma_{2 E}^{(k)} \\
0, \text { elsewhere }
\end{array},\right.
$$

where $\gamma_{D}$, and $\gamma_{2 E}^{(k)}$ denote the instantaneous SNR of the main link $R-D$ and the channel $R-E_{k}$, respectively and are given as

$$
\begin{gathered}
\gamma_{D}=\min \left(\bar{\gamma}_{R}, \frac{\bar{\gamma}_{I}}{g_{R P}}\right) \sum_{t=1}^{L_{D}} g_{R D_{t}}, \\
\gamma_{2 E}^{(k)}=\min \left(\bar{\gamma}_{R}, \frac{\bar{\gamma}_{I}}{g_{R P}}\right) \sum_{u=1}^{L_{E_{k}}} g_{R E_{k}^{(u)}},
\end{gathered}
$$

with $\bar{\gamma}_{R}=P_{R}^{\max } / N_{0}$.

Remark 1. - One can see from (14) and (15), that the PHY layer security at the first hop in the presence of a friendly jammer can be enhanced by increasing separately $\bar{\gamma}_{I}, \bar{\gamma}_{S_{i}}$, or $\bar{\gamma}_{S_{J}}$. Indeed, the increasing scale of the SNR at the relay exceeds the one of the kth eavesdropper as a jamming signal is added to the one received by $E_{k}$. However, in the absence of a friendly jammer, one can see from (14) and (16) that only the impact of legitimate and wiretap channels' parameters can make the distinction between the two associated SNRs. Consequently, the smaller $\lambda_{S_{i} R}$, the greater the secrecy capacity and then the security gets improved.

- From (18) and (19), it can be noticed that increasing either $\bar{\gamma}_{R}$ or $\bar{\gamma}_{I}$ enhances more the capacity of the legitimate link as D performs the MRC technique. Additionally, increasing the number of antennas at the receiver increases the SNR at D. Consequently, the system's security gets enhanced as well.

\section{B. Exact Secrecy Outage Probability}

In this paper, the SOP is chosen as a performance metric and it accounts for the probability that the secrecy capacity is less than a predefined secrecy rate $R_{s}$. For the considered system, the $N$ sources are taking rounds in accessing the spectrum then one jammer is randomly selected among the $N-1$ remaining sources. The SOP if there were no jamming, can be expressed as

$$
S O P=\frac{1}{N} \sum_{i=1}^{N} S O P^{(i)},
$$


while in the presence of a jammer, it becomes [17]

$$
S O P=\frac{1}{N(N-1)} \sum_{i=1}^{N} \sum_{\substack{J=1 \\ J \neq i}}^{N} S O P^{(i, J)},
$$

where $S O P^{(i)}$ and $S O P^{(i, J)}$ account for SOP of the system linking $S_{i}$ with $D$ in the presence of eavesdroppers, and in the absence and presence of the $J$ th friendly jammer, respectively. The SOP of the considered system stands for the probability that at least one of the secrecy capacities falls below a predefined secrecy rate $R_{s}$, namely

$$
\begin{aligned}
S O P^{(i, J)} & =1-\prod_{k=1}^{M} \operatorname{Pr}\left(\min \left(C_{1 S}^{(i, k, J)}, C_{2 S}^{(k)}\right) \geq R_{s}\right) \\
& =1-\prod_{k=1}^{M}\left[1-S O P_{1}^{(i, k, J)}\right]\left[1-S O P_{2}^{(k)}\right]
\end{aligned}
$$

and

$$
S O P^{(i)}=1-\prod_{k=1}^{M}\left[1-S O P_{1}^{(i, k)}\right]\left[1-S O P_{2}^{(k)}\right]
$$

where $S O P_{1}^{(i, k, J)}$ and $S O P_{1}^{(i, k)}$ stand for the secrecy capacities at the first hop in the presence and absence of a friendly jammer, respectively, and $S O P_{2}^{(k)}$ represents the secrecy capacity at the second hop. One can see from (22) and (23) that the computation of SOP requires the knowledge of $S O P_{1}^{(i, k, J)}, S O P_{1}^{(i, k)}$, and $S O P_{2}^{(k)}$ as well.

Remark 2. As $S O P_{1}^{(i, k, J)}, S O P_{1}^{(i)}$, and $S O P_{2}^{(k)}$ are between 0 and 1 , it is worth mentioning that the greater is $M$, the greater is SOP (approaches 1), and then the system becomes vulnerable to eavesdropping attack.

Theorem 1. The closed-form expressions of $S O P_{1}^{(i, k, J)}, S O P_{1}^{(i, k)}$, and $S O P_{2}^{(k)}$ under Nakagami$m$ fading model are given by (24), (25), and (26), respectively, as shown at the top of the next page, where $\varpi_{i}^{(k)}=\gamma \lambda_{S_{i} R}+\lambda_{S_{i} E_{k}}, \sigma_{i}=\bar{\gamma}_{I} / \bar{\gamma}_{S_{i}}, \delta=\bar{\gamma}_{I} / \bar{\gamma}_{R}, \gamma=2^{R_{S}}, \theta_{i}^{(k, J)}=$ $\lambda_{S_{J} E_{k}} /\left(\bar{\gamma}_{S_{J}} \lambda_{S_{i} E_{k}}\right), \varphi_{J}=\lambda_{S_{J} P} \bar{\gamma}_{I} / \bar{\gamma}_{S_{J}}, \varsigma_{i}^{(k, J)}=\lambda_{S_{J} E_{k}} /\left(\lambda_{S_{J} P} \lambda_{S_{i} E_{k}}\right), \varphi_{R}=\lambda_{R P} \bar{\gamma}_{I} / \bar{\gamma}_{R}, \varphi_{S_{i}}=$ $\lambda_{S_{i} P} \bar{\gamma}_{P} / \bar{\gamma}_{S_{i}}$

$$
\mathcal{M}_{1}^{(h, l)}(z)=G_{2,3}^{2,2}\left(\begin{array}{c}
-h, 1 ;- \\
z \\
\mu_{i, k}^{(h, l)}, L_{E_{k}} m_{S_{J} E_{k}} ; 0
\end{array}\right)
$$




$$
\begin{aligned}
S O P_{1}^{(i, k, J)}= & 1-\frac{\gamma\left(m_{S_{i} P}, \varphi_{S_{i}}\right) \Gamma\left(L_{R} m_{S_{i} R}, \frac{\sigma_{i} \xi_{S_{i} R}}{\bar{\gamma}_{I}}\right)+\mathcal{M}_{3}\left(\frac{\xi_{S_{i} R}}{\lambda_{S_{i} P} \bar{\gamma}_{I}}\right)}{\Gamma\left(L_{R} m_{S_{i} R}\right) \Gamma\left(m_{S_{i} P}\right)}+\frac{\gamma \alpha_{i}^{(k, J)}}{\Gamma\left(m_{S_{i} P}\right)} \sum_{h=0}^{L_{E_{k}} m_{S_{i} E_{k}}-1} \Omega_{h}^{(i, k)} \sum_{l=0}^{L_{R} m_{S_{i} R}-1} \\
& \times\left[\frac{\gamma\left(m_{S_{i} P}, \varphi_{S_{i}}\right) e^{-\frac{\sigma_{i} \xi_{S_{i} R}}{\bar{\gamma}_{I}}}}{\sigma_{i}^{-L_{R} m_{S_{i} R}+l+1}}+\frac{\lambda_{S_{i} P}^{m_{S_{i} P} P} \Gamma\left(m_{S_{i} P}+L_{R} m_{S_{i} R}-l-1, \varphi_{S_{i}}+\frac{\sigma_{i} \xi_{S_{i} R}}{\bar{\gamma}_{I}}\right)}{\left(\lambda_{S_{i} P}+\frac{\xi_{S_{i} R}}{\bar{\gamma}_{I}}\right)^{m_{S_{i} P}+L_{R} m_{S_{i} R}-l-1}}\right] \\
& \times\left[\gamma\left(m_{S_{J} P}, \varphi_{J}\right) \mathcal{M}_{1}^{(h, l)}\left(\varpi_{i}^{(k)} \theta_{i}^{(k, J)}\right)+\mathcal{M}_{2}^{(h, l)}\left(\frac{\varsigma_{i}^{(k, J)} \varpi_{i}^{(k)}}{\bar{\gamma}_{I}}\right)\right]
\end{aligned}
$$

$$
\begin{aligned}
S O P_{1}^{(i, k)}= & 1-\frac{\lambda_{S_{i} R}^{L_{R} m_{S_{i} R}} \gamma}{\Gamma\left(L_{E_{k}} m_{S_{i} E_{k}}\right) \Gamma\left(L_{R} m_{S_{i} R}\right) \Gamma\left(m_{S_{i} P}\right)} \sum_{l=0}^{L_{R} m_{S_{i} R}-1} \frac{\Upsilon_{l}^{(i)} \bar{\gamma}_{I}^{-L_{R} m_{S_{i} R}+l+1}}{\left(\gamma \lambda_{S_{i} R}\right)^{l+1}} G_{2,2}^{1,2}\left(\left.\frac{\lambda_{S_{i} E_{k}}}{\lambda_{S_{i} R} \gamma}\right|_{L_{E_{k}} m_{S_{i} E_{k}}} ^{-l, 0}\right. \\
& \times\left[\frac{\gamma\left(m_{S_{i} P}, \varphi_{S_{i}}\right) e^{-\frac{\sigma_{i} \xi_{S_{i} R}}{\bar{\gamma}_{I}}}}{\sigma_{i}^{-L_{R} m_{S_{i} R}+l+1}}+\frac{\lambda_{S_{i} P}^{m_{S_{i} P} P} \Gamma\left(m_{S_{i} P}+L_{R} m_{S_{i} R}-l-1, \varphi_{S_{i}}+\frac{\sigma_{i} \xi_{S_{i} R}}{\bar{\gamma}_{I}}\right)}{\left(\lambda_{S_{i} P}+\frac{\xi_{S_{i} R}}{\bar{\gamma}_{I}}\right)^{m_{S_{i} P}+L_{R} m_{S_{i} R}-l-1}}\right]
\end{aligned}
$$

$$
\begin{aligned}
& \mathcal{M}_{2}^{(h, l)}(z)=G_{3,3}^{2,3}\left(z \mid \begin{array}{c}
\left(1-m_{S_{J} P}, \varphi_{J}\right),(1,0),(-h, 0) ;- \\
\left(L_{E_{k}} m_{S_{J} E_{k}}, 0\right),\left(\mu_{i, k}^{(h, l)}, 0\right) ;(0,0)
\end{array}\right), \\
& \mu_{i, k}^{(h, l)}=L_{E_{k}} m_{S_{i} E_{k}}-h+l, \\
& \xi_{v}=\lambda_{v}(\gamma-1) ; v=\left\{S_{i} R, R D\right\}, \\
& \mathcal{M}_{3}(z)=G_{2,2}^{2,1}\left(\begin{array}{l|c}
z & \begin{array}{c}
\left(1-m_{S_{i} P}, \varphi_{S_{i}}\right) ;(1,0) \\
(0,0),\left(L_{R} m_{S_{i} R}, 0\right) ;-
\end{array}
\end{array}\right) \text {, } \\
& \Omega_{h}^{(i, k)}=\left(\begin{array}{c}
L_{E_{k}} m_{S_{i} E_{k}}-1 \\
h
\end{array}\right) \lambda_{S_{i} E_{k}}^{L_{E_{k}} m_{S_{i} E_{k}}-h-1}, \\
& \alpha_{i}^{(k, J)}=\frac{\beta_{k}^{(J)} \lambda_{S_{i} R}^{L_{R} m_{S_{i} R}}}{\Gamma\left(L_{E_{k}} m_{S_{i} E_{k}}\right) \Gamma\left(L_{R} m_{S_{i} R}\right)}, \\
& \beta_{k}^{(J)}=\frac{1}{\Gamma\left(L_{E_{k}} m_{S_{J} E_{k}}\right) \Gamma\left(m_{S_{J} P}\right)}, \\
& \Upsilon_{l}^{(i)}=\left(\begin{array}{c}
L_{R} m_{S_{i} R}-1 \\
l
\end{array}\right) \gamma^{l}(\gamma-1)^{L_{R} m_{S_{i} R}-1-l},
\end{aligned}
$$




$$
\begin{aligned}
S O P_{2}^{(k)}= & 1-\frac{\lambda_{R D}^{L_{D} m_{R D}}}{\Gamma\left(L_{E_{k}} m_{R E_{k}}\right) \Gamma\left(L_{D} m_{R D}\right) \Gamma\left(m_{R P}\right)} \sum_{j=0}^{L_{D} m_{R D}-1} \frac{\mathcal{B}_{j} \bar{\gamma}_{I}^{-L_{D} m_{R D}+j+1}}{\lambda_{R D}^{j+1}} G_{2,2}^{1,2}\left(\frac{\lambda_{R E_{k}}}{\lambda_{R D} \gamma} \mid \begin{array}{c}
-j, 1 ;- \\
L_{E_{k}} m_{R E_{k}} ; 0
\end{array}\right) \\
& \times\left[\frac{e^{-\frac{\delta \xi_{R D}}{\bar{\gamma}_{I}}} \gamma\left(m_{R P}, \varphi_{R}\right)}{\delta^{-L_{D} m_{R D}+j+1}}+\frac{\lambda_{R P}^{m_{R P}} \Gamma\left(L_{D} m_{R D}+m_{R P}-j-1, \varphi_{R}+\frac{\delta \xi_{R D}}{\bar{\gamma}_{I}}\right)}{\left(\frac{\xi_{R D}}{\bar{\gamma}_{I}}+\lambda_{R P}\right)^{L_{D} m_{R D}+m_{R P}-j-1}}\right]
\end{aligned}
$$

$$
\mathcal{B}_{j}=\left(\begin{array}{c}
L_{D} m_{R D}-1 \\
j
\end{array}\right)(\gamma-1)^{L_{D} m_{R D}-1-j},
$$

where $G_{p, q}^{m, n}\left(z \mid \begin{array}{c}\left(a_{l}\right)_{l \leq p} \\ \left(b_{u}\right)_{u \leq q}\end{array}\right)$ denotes the Meijer's G-function [21. Eq. (9.301)], $G_{p, q}^{m, n}\left(z \mid \begin{array}{c}\left(a_{l}, b_{l}\right)_{l \leq p} \\ \left(c_{u}, d_{u}\right)_{u \leq q}\end{array}\right)$ accounts for the upper incomplete Meijer's G-function [22, Eq. (1.1.1)], and $\Gamma(.,$.$) denotes the$ upper incomplete Gamma function [21, Eq. (8.350.2)].

Proof: The proof is provided in Appendix A.

\section{Asymptotic Secrecy Outage Probability}

In this subsection, we provide an asymptotic analysis of the derived closed-form expressions of the SOP. The expressions given in (24), 25], and 26) can be approximated for SNR regime by considering $\bar{\gamma}_{P} \rightarrow \infty$.

Theorem 2. The Asymptotic expression of the SOP in the absence of a jammer is given by (50) as shown in the next page, while it is expressed in the presence of a jamming signal depending on various cases as follows

- $L_{R} m_{S_{i} R}<L_{E_{k}} m_{S_{J} E_{k}}$

$$
\begin{aligned}
S O P^{(i, k, J)} \sim & 1-\prod_{k=1}^{M} \mathcal{A}_{R E_{k}, R D, D}(1) \\
& -\frac{\sum_{k=1}^{M} \prod_{\substack{j=1 \\
j \neq k}}^{M} \mathcal{A}_{R E_{j}, R D, D}(1)}{\bar{\gamma}_{I}} \\
& \times \mathcal{A}_{R E_{k}, R D, R, D}\left(1-\mathcal{P}\left(S_{i} R\right) \mathcal{C}_{1}^{(i, k, J)}\right),
\end{aligned}
$$




$$
\begin{aligned}
S O P^{(i)} \sim & 1-\prod_{k=1}^{M} \mathcal{A}_{R E_{k}, R D, D}(1) \mathcal{A}_{S_{i} E_{k}, S_{i} R, R}(1)-\frac{1}{\bar{\gamma}_{I}} \sum_{\substack{k=1 \\
j}}^{M} \prod_{\substack{j=1 \\
j \neq k}}^{M} \mathcal{A}_{R E_{j}, R D, D}(1) \mathcal{A}_{S_{i} E_{j}, S_{i} R, R}(\text { \$D) }) \\
& \times\left(\mathcal{A}_{R E_{k}, R D, D}(1) \mathcal{A}_{S_{i} E_{k}, S_{i} R, S_{i}, R}+\mathcal{A}_{S_{i} E_{k}, S_{i} R, R}(1) \mathcal{A}_{R E_{k}, R D, R, D}\right)
\end{aligned}
$$

- $L_{R} m_{S_{i} R}>L_{E_{k}} m_{S_{J} E_{k}}$

$$
\begin{aligned}
S O P^{(i, k, J)} \sim & 1-\prod_{k=1}^{M} \mathcal{A}_{R E_{k}, R D, D}(1) \\
& -\frac{\sum_{k=1}^{M} \prod_{\substack{j=1 \\
j \neq k}}^{M} \mathcal{A}_{R E_{j}, R D, D}(1)}{\bar{\gamma}_{I}} \\
& \times \mathcal{A}_{R E_{k}, R D, R, D}\left(1-\mathcal{P}\left(S_{J} E_{k}\right) \mathcal{C}_{2}^{(i, k, J)}\right),
\end{aligned}
$$

- $L_{R} m_{S_{i} R}=L_{E_{k}} m_{S_{J} E_{k}}=1$

$$
\begin{aligned}
S O P^{(i, J)} & \sim 1-\prod_{k=1}^{M} \mathcal{A}_{R E_{k}, R D, D}(1)+\frac{\log \left(\bar{\gamma}_{I}\right)}{\bar{\gamma}_{I}} \\
& \times \sum_{k=1}^{M} \prod_{\substack{j=1 \\
j \neq k}}^{M} \mathcal{A}_{R E_{j}, R D, D}(1) \mathcal{A}_{R E_{k}, R D, D}(1) \mathcal{C}_{3}^{(i, k, J)} .
\end{aligned}
$$

- $L_{R} m_{S_{i} R}=L_{E_{k}} m_{S_{J} E_{k}}$ and $L_{E_{k}} m_{S_{J} E_{k}}>1$

$$
\begin{aligned}
S O P^{(i, J)} & \sim 1-\prod_{k=1}^{M} \mathcal{A}_{R E_{k}, R D, D}(1)-\frac{1}{\bar{\gamma}_{I}} \sum_{\substack { k=1 \\
\begin{subarray}{c}{j=1 \\
j \neq k{ k = 1 \\
\begin{subarray} { c } { j = 1 \\
j \neq k } }\end{subarray}}^{M} \\
& \times \mathcal{A}_{R E_{j}, R D, D}(1) \mathcal{A}_{R E_{k}, R D, R, D},
\end{aligned}
$$

where $\mathcal{P}(q)=1-\operatorname{sgn}\left(L_{v} m_{q}-1\right), q=\left\{S_{i} R, S_{J} E_{k}\right\}$, sgn stands for sign function

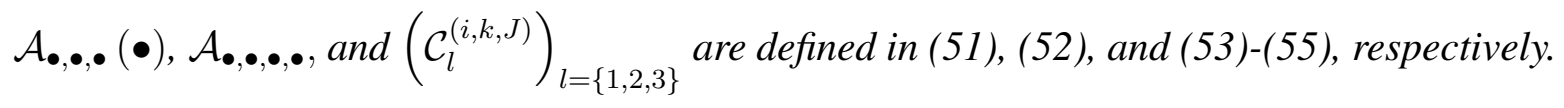

Proof: The proof is provided in Appendix B.

\section{RESULTS AND DISCUSSION}

In this section, we validate the derived analytical results through Monte Carlo simulation by generating $10^{6}$ Gamma-distributed random variables. The setting parameters of the simulation 


$$
\mathcal{A}_{e, c, v}(y)=\frac{1}{\Gamma\left(L_{v} m_{e}\right) \Gamma\left(m_{c}\right)} G_{2,2}^{1,2}\left(\frac{\lambda_{e}}{\lambda_{c} \gamma} \mid \begin{array}{c}
-L_{v} m_{c}+y, 1 ;- \\
L_{v} m_{e} ; 0
\end{array}\right), e=\left\{S_{i} E_{k}, R E_{k}\right\}, c=\left\{S_{i} R, R D\right\}, y=\{1,2\}
$$

$$
\mathcal{A}_{e, c, u, v}=\left\{\begin{array}{c}
\frac{\lambda_{c}\left(L_{v} m_{c}-1\right) \mathcal{A}_{e, c, v}(2)}{\Gamma\left(m_{u P}\right)}\left[\delta \gamma\left(m_{u P}, \varphi_{u}\right)+\frac{\Gamma\left(m_{u P}+1, \varphi_{u}\right)}{\lambda_{u P}}\right] \\
-\frac{\xi_{c} \mathcal{A}_{e, c, v}(1)}{\Gamma\left(m_{u P}\right)}\left[\delta\left(\gamma\left(m_{u P}, \varphi_{u}\right)+\varphi_{R}^{m_{u P}-1} e^{-\varphi_{u}}\right)+\frac{m_{u P}}{\lambda_{u P}} \Gamma\left(m_{u P}, \varphi_{u}\right)\right]
\end{array}\right\}, u=\left\{S_{i}, R\right\}, v=\{R, D\}
$$

are summarized in Table 1. Indeed, the values of fading severity parameter $m_{\bullet}$ have been chosen such that the wiretap channel is better than the legitimate one. Moreover, their values are taken integer in the range 2.5 similarly to [6] and [20]. On the other hand, the average SNR, which is inversely proportional to $\lambda_{\boldsymbol{0}}$, the legitimate link is considered better than the one of the wiretap channel. It is worthwhile that these parameters are associated with all figures except those indicating other values. As one can see in Figs. 2-5, all closed-form and simulation curves are perfectly matched for considered parameters' values.

Fig. 2 and Fig. 3 depict closed-form and asymptotic expressions for the SOP versus $\bar{\gamma}_{I}$ for various values of antennas' numbers in both the presence and absence of a friendly jammer cases, respectively. As stated in remark 1 , It can be noticed that the greater $\bar{\gamma}_{I}$, the smaller the SOP. Interestingly, above a certain threshold of $\bar{\gamma}_{I}$ the SOP becomes steady this can be obviously justified from (8) and (9) that above that threshold, both sources and relay will always transmit with their maximum powers. Consequently, the legitimate and wiretap capacities of each hop

TABLE I: Simulation parameters.

\begin{tabular}{c|c|c|c|c|c}
\hline \hline Parameter & $M$ & $N$ & $\lambda_{S_{i} R}$ & $\lambda_{S_{i} P}$ & $\lambda_{S_{i} E_{k}}$ \\
\hline value & 3 & 4 & 0.1 & 0.3 & 0.6 \\
\hline Parameter & $\lambda_{R E_{k}}$ & $\lambda_{R P}$ & $\lambda_{R D}$ & $m_{S_{i} R}$ & $m_{S_{i} P}$ \\
\hline value & 0.6 & 0.2 & 0.1 & 2 & 3 \\
\hline Parameter & $m_{S_{i} E_{k}}$ & $m_{R D}$ & $m_{R E_{k}}$ & $m_{R P}$ & \\
\hline value & 5 & 2 & 4 & 3 & \\
\hline \hline
\end{tabular}




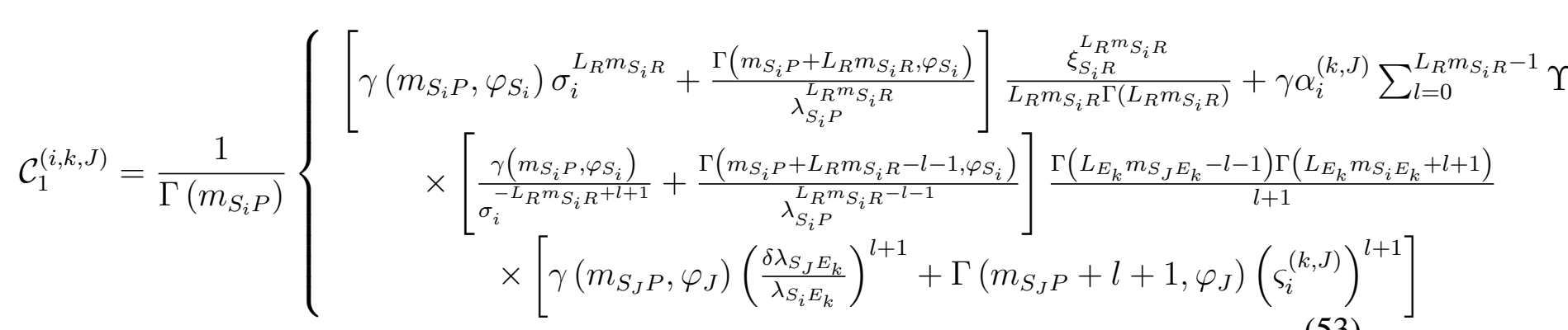

$$
\begin{aligned}
& \mathcal{C}_{2}^{(i, k, J)} \sim \frac{\gamma^{L_{R} m_{S_{i} R}} \alpha_{i}^{(k, J)}}{L_{E_{k}} m_{S_{J} E_{k}}}\left[\gamma\left(m_{S_{J} P}, \varphi_{J}\right)\left(\frac{\delta \lambda_{S_{J} E_{k}}}{\lambda_{S_{i} E_{k}}}\right)^{L_{E_{k}} m_{S_{J} E_{k}}}+\Gamma\left(m_{S_{J} P}+L_{E_{k}} m_{S_{J} E_{k}}, \varphi_{J}\right)\left(\varsigma_{i}^{(k, J)}\right)^{L_{E_{k}} m_{S_{J} E^{E}}}{ }^{(54}\right] \\
& \times \sum_{h=0}^{L_{E_{k}} m_{S_{i} E_{k}}-1} \frac{\Omega_{h}^{(i, k)} \Gamma\left(L_{E_{k}} m_{S_{i} E_{k}}+L_{R} m_{S_{i} R}-L_{E_{k}} m_{S_{J} E_{k}}-h-1\right) \Gamma\left(L_{E_{k}} m_{S_{J} E_{k}}+h+1\right)}{\left(\varpi_{i}^{(k)}\right)^{L_{E_{k}} m_{S_{i} E_{k}}+L_{R} m_{S_{i} R}-L_{E_{k}} m_{S_{J} E_{k}}-h-1}},
\end{aligned}
$$

remain constant, leading to a constant value of SOP. Interestingly, by comparing the SOP values in the two aforementioned figures, one can ascertain that better secrecy is achieved by using a friendly jammer. In addition, the asymptotic curves are plotted under the considered fading severity values (i.e., $m_{S_{i} R}=2, m_{S_{J} E_{k}}=5$ ) from Eqs. (37), (51)-(53). Clearly, the asymptotic curves match with the closed-form ones in high SNR regime.

Fig. 4 illustrates the SOP versus $\bar{\gamma}_{S_{J}}$ for numerous values of branches' number $L_{D}$ at the receiver $D$. Again, as indicated in remark 1, one can realize that the higher $\bar{\gamma}_{S_{J}}$ and $L_{D}$, the smaller the SOP and therefore the system's security gets improved.

Fig. 5 and Fig. 6 show the SOP as a function of the number of eavesdroppers $M$ for different values of $\bar{\gamma}_{S_{J}}$ and by considering both cases i.e., presence and absence of jammer. One can observe that the smaller $\bar{\gamma}_{S_{J}}$ or the greater $M$ the worst is the system's secrecy as highlighted in remark 1 and 2, respectively. In addition, introducing a jamming signal improves significantly the secrecy performance for high values of $\bar{\gamma}_{S_{J}}$ or in the presence of small numbers of eavesdroppers. Fig. 7 depicts the SOP as a function of the number of eavesdroppers by considering the presence and absence of a friendly jammer. It is worth mentioning that better security is obviously achieved for the case of presence of jammer and multi-antenna nodes, while the scenario of the absence of jammer and legitimate nodes equipped with a single antenna is the worst case. For this reason, our 


$$
\begin{aligned}
\mathcal{C}_{3}^{(i, k, J)=} & \frac{\gamma^{L_{R} m_{S_{i} R} \alpha_{i}}(k, J)}{\varpi^{L_{R} m_{S_{i} R}-L_{E_{k}} m_{S_{J} E_{k}}}}\left[\gamma\left(m_{S_{J} P}, \varphi_{J}\right)\left(\frac{\delta \lambda_{S_{J} E_{k}}}{\lambda_{S_{i} E_{k}}}\right)^{L_{E_{k}} m_{S_{J} E_{k}}}+\left(\varsigma_{i}^{(k, J)}\right)^{L_{E_{k}} m_{S_{J} E_{k}}} \Gamma\left(m_{S_{J} P}+L_{E_{k}} m_{S_{J} E_{k}},\right.\right. \\
& \frac{(-1)^{L_{E_{k}} m_{S_{J} E_{k}}-L_{R} m_{S_{i} R}} \Gamma\left(L_{E_{k}} m_{S_{i} E_{k}}+L_{E_{k}} m_{S_{J} E_{k}}\right)}{L_{E_{k}} m_{S_{J} E_{k}}}
\end{aligned}
$$

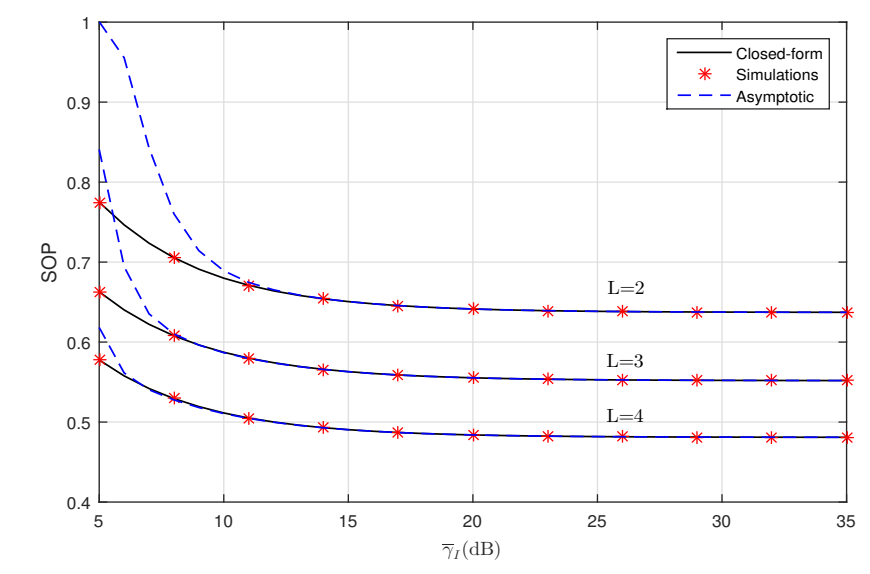

Fig. 2: SOP vs $\bar{\gamma}_{I}$ for different values of antennas at the destination in the presence of a friendly jammer for $\eta=\sigma_{i}=\delta=0.1$ and $L_{R}=L_{E_{k}}=L_{D}=L$.

aim here is to investigate if the security gets enhanced when having artificial noise and legitimate nodes with a single antenna or the scenario of the absence of jammer and all legitimate nodes are equipped with multiple antennas. One can obviously notice that the system's security is improved when diversity is used at the legitimate nodes. Additionally, in the presence of an important number of eavesdroppers, the friendly jammer does not contribute to the enhancement of the system's security.

\section{Conclusions}

In this paper, the physical layer security of a dual-hop underlay uplink CRN operating under Nakagami- $m$ fading channels was investigated. We considered multiple sources communicating, in turn, with the base station through a relay in the presence of several eavesdroppers attempting to overhear the communication channels. All receivers, i.e., legitimates and wiretappers, were assumed to be equipped with multiple antennas and perform the MRC technique. Closed-form and asymptotic expressions for the SOP under various cases of fading parameters' values were 


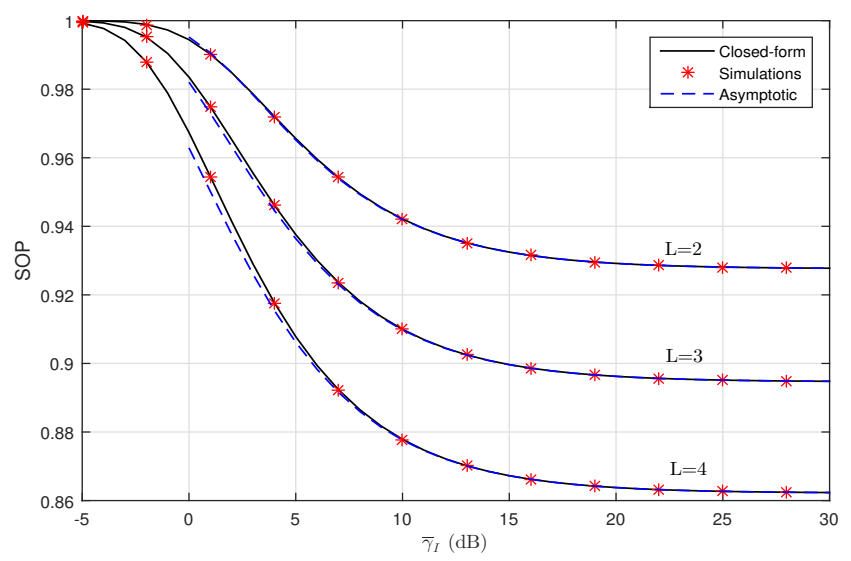

Fig. 3: SOP vs $\bar{\gamma}_{I}$ in the absence of a friendly jammer for $\sigma_{i}=\delta=0.1$ and $L_{R}=L_{E_{k}}=L_{D}=L$.

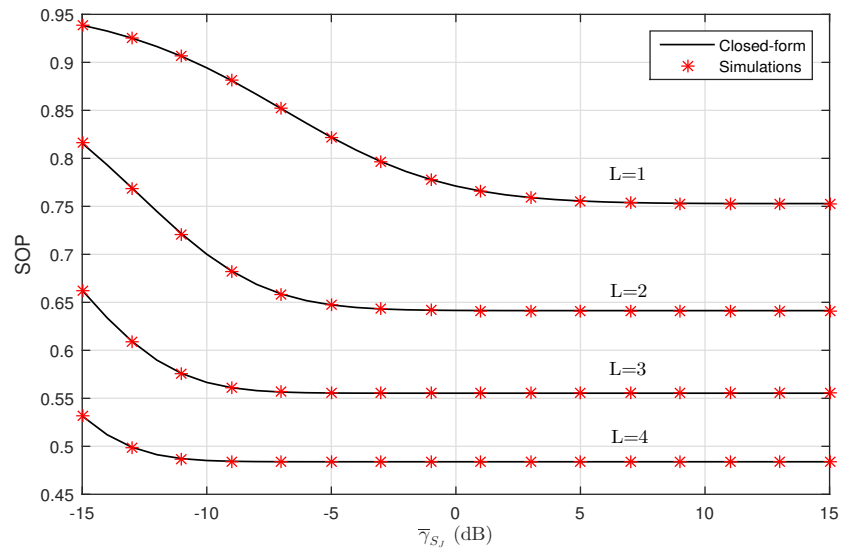

Fig. 4: SOP vs $\bar{\gamma}_{S_{J}}$ for different values of antennas at the destination for $\bar{\gamma}_{I}=\bar{\gamma}_{S_{i}}=\bar{\gamma}_{R}=20$ $\mathrm{dB}$ and $L_{R}=L_{E_{k}}=L_{D}=L$.

derived by considering two scenarios namely, (i) presence and (ii) absence of a friendly jammer. The obtained results showed that the best secrecy is achieved in the presence of a small number of eavesdroppers when increasing the transmit power of the SUs', the number of antennas at the legitimate receiver and the maximum tolerated interference power at the PU as well. Interestingly, we showed that equipping the legitimate nodes by multiple antennas leads to a noticeable enhancement of the system's security rather than sending an artificial noise. As future work, we intend to investigate the impact of NOMA jointly with the key parameters considered in this work on the system's secrecy. We also intend to consider the case of amplify-and-forward 


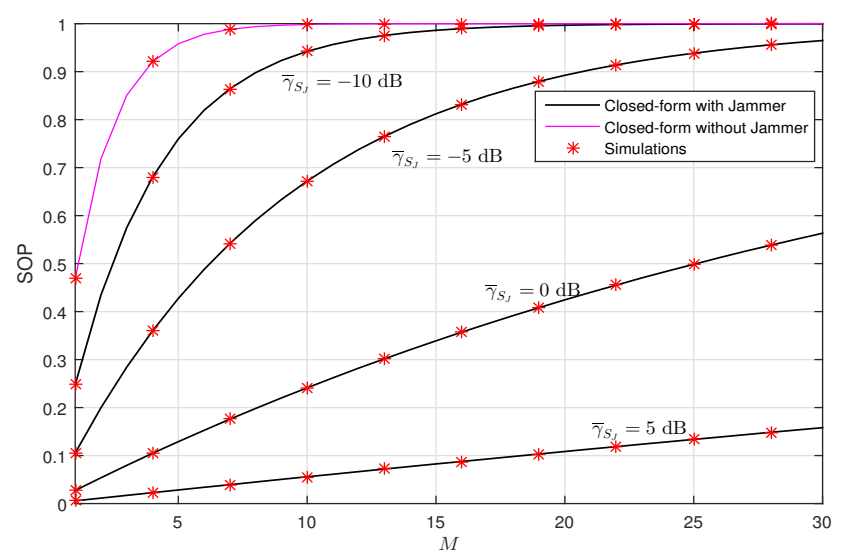

Fig. 5: SOP vs number of eavesdroppers and different values $\bar{\gamma}_{S_{J}}$ for $L_{D}=4$ and $\bar{\gamma}_{I}=\bar{\gamma}_{S_{i}}=$ $\bar{\gamma}_{R}=20 \mathrm{~dB}$.

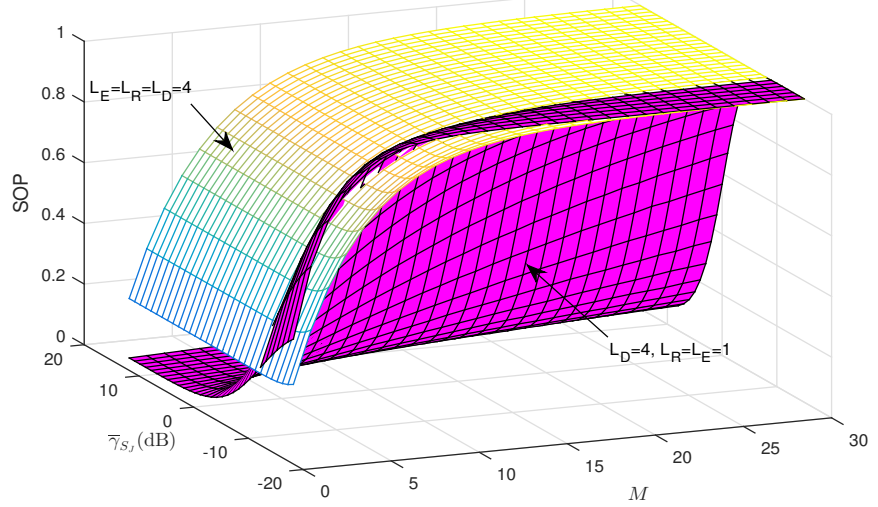

Fig. 6: SOP vs number of eavesdroppers and $\bar{\gamma}_{S_{J}}$ for $\bar{\gamma}_{I}=\bar{\gamma}_{S_{i}}=\bar{\gamma}_{R}=20 \mathrm{~dB}$.

relaying protocol and investigate the impact of numerous jammer selection policies on the overall system's security.

\section{APPENDIX A: PROOF OF THEOREM 1}

\section{A. Expression of SOP at the First Hop}

The SOP at the first hop in the absence and presence of a friendly jammer is given, respectively, by

$$
S O P_{1}^{(i, k)}=1-\gamma \int_{x=0}^{\infty} f_{g_{S_{i} P}}(x) \Xi_{2}^{(i, k)}(x) d x
$$




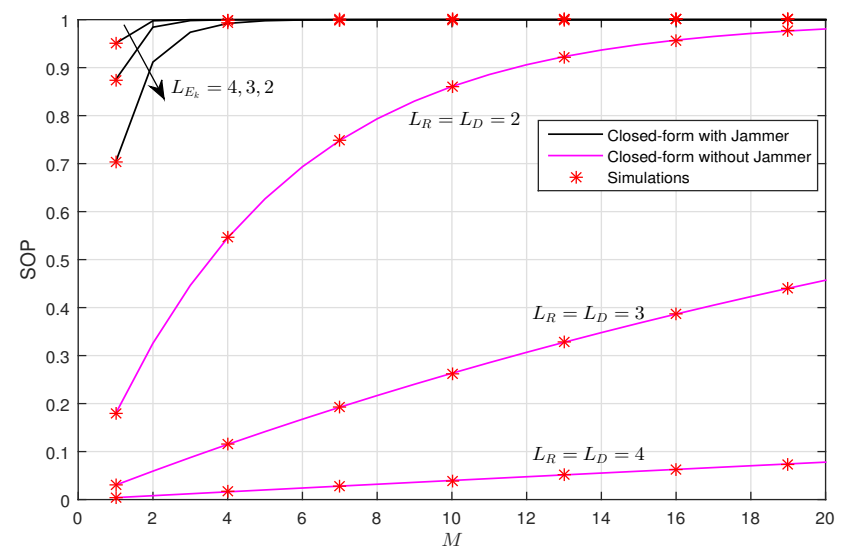

Fig. 7: SOP vs the number of eavesdroppers in the presence and absence of friendly jammer and different numbers of antennas for $\bar{\gamma}_{S_{J}}=20 \mathrm{~dB}$.

$$
S O P_{1}^{(i, k, J)}=1-\gamma \int_{x=0}^{\infty} f_{g_{S_{i} P}}(x) \Xi_{1}^{(i, k, J)}(x) d x
$$

where (57) and (56) hold by using integration by parts on [23, Eq. (33)], with

$$
\Xi_{1}^{(i, k, J)}(x)=\int_{0}^{\infty} f_{\gamma_{R}^{(i)} \mid g_{S_{i} P}=x}(\gamma y+\gamma-1) F_{\gamma_{1 E}^{(i, k, J)} \mid g_{S_{i} P}=x}(y) d y
$$

and

$$
\Xi_{2}^{(i, k)}(x)=\int_{0}^{\infty} f_{\gamma_{R}^{(i)} \mid g_{S_{i} P}=x}(\gamma y+\gamma-1) F_{\gamma_{1 E}^{(i, k)} \mid g_{S_{i} P}=x}(y) d y,
$$

and $\gamma$ is being defined in Theorem 1 .

- Conditional CDF of $\gamma_{R}^{(i)}$

The CDF of $\gamma_{R}^{(i)}$ for a given $g_{S_{i} P}$ can be expressed as

$$
\begin{aligned}
F_{\gamma_{R}^{(i)} \mid g_{S_{i} P}=x}(z) & =\operatorname{Pr}\left(\min \left(\bar{\gamma}_{S_{i}}, \frac{\bar{\gamma}_{I}}{x}\right) \mathcal{E}_{S_{i} R} \leq z\right) \\
& =F_{\mathcal{E}_{S_{i} R}}\left(\frac{z}{\Phi(x)}\right)
\end{aligned}
$$

where $\mathcal{E}_{S_{i} R}=\sum_{u=1}^{L_{R}} g_{S_{i} R_{u}}, \Phi(x)=\bar{\gamma}_{S_{i}}$ for $x \leq \bar{\gamma}_{I} / \bar{\gamma}_{S_{i}}$ and $\Phi(x)=\bar{\gamma}_{I} / x$ for $x>\bar{\gamma}_{I} / \bar{\gamma}_{S_{i}}$.

1) SOP at the First Hop with the Absence of a Jamming Signal: The conditional CDF of $\gamma_{1 E}^{(i, k)}$ can be expressed as

$$
\begin{aligned}
F_{\gamma_{1 E}^{(i, k)} \mid g_{S_{i} P}=x}(y) & =\operatorname{Pr}\left(\min \left(\bar{\gamma}_{S_{i}}, \frac{\bar{\gamma}_{I}}{x}\right) \mathcal{E}_{S_{i} E_{k}} \leq y\right) \\
& =F_{\mathcal{E}_{S_{i} E_{k}}}\left(\frac{y}{\Phi(x)}\right) .
\end{aligned}
$$


where $\mathcal{E}_{S_{i} E_{k}}=\sum_{u=1}^{L_{R}} g_{S_{i} E_{k}^{(u)}}$.

It is worth mentioning that for i.i.d Nakagami-m channels, $\mathcal{E}_{S_{i} R}$ and $\mathcal{E}_{S_{i} E_{k}}$ are Gamma distributed with shape and scale parameters $L_{R} m_{S_{i} R}$ and $\lambda_{S_{i} R}, L_{E_{k}} m_{S_{i} E_{k}}$ and $\lambda_{S_{i} E_{k}}$, respectively.

Substituting (60) and (61) into (59), and using [24, Eqs. (06.06.26.0004.01), (07.34.21.0088.01] yields

$$
\begin{aligned}
\Xi_{2}^{(i, k)}(x) & =\frac{\lambda_{S_{i} R}^{L_{R} m_{S_{i} R}} e^{-\frac{\xi_{S_{i} R}}{\Phi(x)}}}{\Phi^{L_{R} m_{S_{i} R}}(x) \Gamma\left(L_{E_{k}} m_{S_{i} E_{k}}\right) \Gamma\left(L_{R} m_{S_{i} R}\right)} \sum_{l=0}^{L_{R} m_{S_{i} R}-1} \Upsilon_{l}^{(i)} \\
& \times\left(\frac{\lambda_{S_{i} R} \gamma}{\Phi(x)}\right)^{-l-1} G_{2,2}^{1,2}\left(\frac{\lambda_{S_{i} E_{k}}}{\lambda_{S_{i} R} \gamma} \mid \begin{array}{c}
-l, 1 ;- \\
L_{E_{k}} m_{S_{i} E_{k}} ; 0
\end{array}\right),
\end{aligned}
$$

where $\Upsilon_{l}^{(i)}$ is defined in $(35)$.

Now, replacing (62) into (56), one can obtain

$$
\begin{aligned}
S_{O} P_{1}^{(i, k)} & =1-\frac{\lambda_{S_{i} R}^{L_{R} m_{S_{i} R}} \gamma}{\Gamma\left(L_{E_{k}} m_{S_{i} E_{k}}\right) \Gamma\left(L_{R} m_{S_{i} R}\right)} \sum_{l=0}^{L_{R} m_{S_{i} R}-1} \frac{\Upsilon_{l}^{(i)}}{\left(\gamma \lambda_{S_{i} R}\right)^{l+1}} \\
& \times \mathcal{H}_{1} G_{2,2}^{1,2}\left(\frac{\lambda_{S_{i} E_{k}}}{\lambda_{S_{i} R} \gamma} \mid \begin{array}{c}
-l, 1 ;- \\
L_{E_{k}} m_{S_{i} E_{k}} ; 0
\end{array}\right),
\end{aligned}
$$

where

$$
\begin{aligned}
& \mathcal{H}_{1}=\int_{0}^{\infty} \frac{f_{g_{S_{i} P}}(x) e^{-\frac{\xi_{S_{i} R}}{\Phi(x)}}}{\Phi^{L_{R} m_{S_{i} R}-l-1}(x)} d x
\end{aligned}
$$

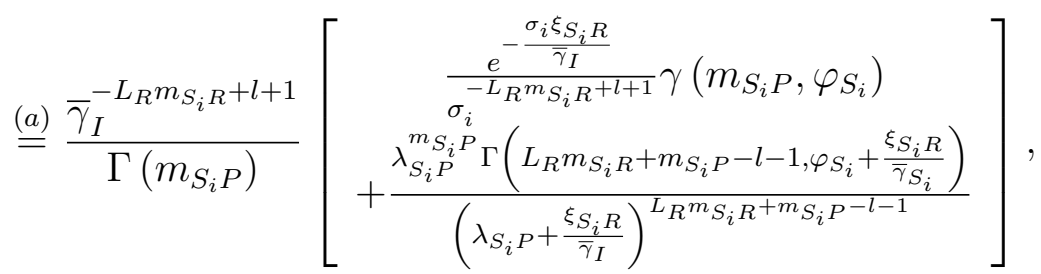

where step $(a)$ is obtained by replacing $\Phi(x)$ by its values, and performing some algebraic manipulations.

Now, incorporating (64) into (63), (25) is attained. 
2) SOP at the First Hop in the Presence of a Jamming Signal: In the presence of a friendly jammer, the CDF of $\gamma_{1 E}^{(i, k, J)}$ for a given $g_{S_{i} P}$ is given by

$$
\begin{aligned}
& F_{\gamma_{1 E}^{(i, k, J)} \mid g_{S_{i} P}=x}(y) \stackrel{(a)}{=} \int_{0}^{\infty} F_{\mathcal{E}_{S_{i} E_{k}}}\left(\frac{y(t+1)}{\Phi(x)}\right) \\
& \times f_{W_{k}^{(J)}}(t) d t \\
& \stackrel{(b)}{=} 1-\Psi_{i}^{(k)}(y) \\
& \times \sum_{h=0}^{L_{E_{k}} m_{S_{i} E_{k}}-1}\left(\begin{array}{c}
L_{E_{k}} m_{S_{i} E_{k}}-1 \\
h
\end{array}\right) \mathcal{V}^{(h)}(y) \text {, }
\end{aligned}
$$

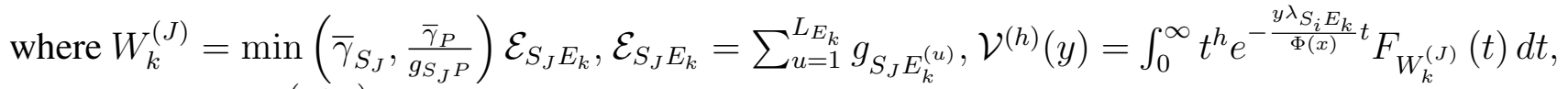
$\Psi_{i}^{(k)}(y)=\frac{y f_{S_{i} E_{k}}\left(\frac{y}{\Phi(x)}\right)}{\Phi(x)}$. Here step 65a holds using the definition 15 , while step $65 \mathrm{~b}$ ) is obtained by using integration by parts alongside the Binomial formula for a positive integer $L_{E_{k}} m_{S_{i} E_{k}}$. Importantly, the derivation of the $\mathrm{CDF}$ of $\gamma_{1 E}^{(i, k, J)}$ requires the one of $W_{k}^{(J)}$, given as

$$
\begin{aligned}
& F_{W_{k}^{(J)}}(t)=\operatorname{Pr}\left(\mathcal{E}_{S_{J} E_{k}} \leq \frac{t}{\bar{\gamma}_{S_{J}}}, \frac{\bar{\gamma}_{I}}{g_{S_{J} P}} \geq \bar{\gamma}_{S_{J}}\right) \\
& +\operatorname{Pr}\left(\frac{\mathcal{E}_{S_{J} E_{k}}}{g_{S_{J} P}} \leq \frac{t}{\bar{\gamma}_{I}}, \frac{\bar{\gamma}_{I}}{g_{S_{J} P}} \leq \bar{\gamma}_{S_{J}}\right) \\
& =F_{\mathcal{E}_{S_{J} E_{k}}}\left(\frac{t}{\bar{\gamma}_{S_{J}}}\right) F_{g_{S_{J} P}}\left(\frac{\bar{\gamma}_{I}}{\bar{\gamma}_{S_{J}}}\right)+\mathcal{I}_{1}^{(k, J)} \text {. }
\end{aligned}
$$

where

$$
\begin{aligned}
\mathcal{I}_{1}^{(k, J)} & =\int_{\frac{\bar{\gamma}_{I}}{\bar{\gamma}_{J}}}^{\infty} f_{g_{S_{J} P}}(\nu) F_{\mathcal{E}_{S_{J} E_{k}}}\left(\frac{t}{\bar{\gamma}_{I}} \nu\right) d \nu \\
& \stackrel{(a)}{=} \int_{\mathcal{L}_{1}} \frac{\Gamma\left(m_{S_{J} P}-s, \varphi_{J}\right) \Gamma\left(L_{E_{k}} m_{S_{J} E_{k}}+s\right) \Gamma(-s)}{2 \pi j \Gamma(1-s)(\kappa t)^{s}\left(\beta_{k}^{(J)}\right)^{-1}} d s \\
& =\beta_{k}^{(J)} \Delta_{k}^{(J)}(t)
\end{aligned}
$$

where $j=\sqrt{-1}, \mathcal{L}_{1}$ is a vertical line of integration chosen such as to separate the left poles of the above integrand function from the right ones, $\Delta_{k}^{(J)}(t)=G_{2,2}^{1,2}\left(\kappa t \mid \begin{array}{c}\left(1-m_{S_{J} P}, \varphi_{J}\right),(1,0) ;- \\ \left(L_{E_{k}} m_{S_{J} E_{k}}, 0\right) ;(0,0)\end{array}\right)$, $\kappa=\lambda_{S_{J} E_{k}} / \lambda_{S_{J} P} \bar{\gamma}_{I}, \varphi_{J}$, and $\beta_{k}^{(J)}$ are defined in Theorem 1 and 34 , respectively. Step $(a)$ holds using [24, Eq. (06.06.26.0004.01)] alongside with (1) and (2). As mentioned above, $\mathcal{E}_{S_{J} E_{k}}$ is also Gamma distributed with parameters $L_{E_{k}} m_{S_{J} E_{k}}$ and $\lambda_{S_{J} E_{k}}$. 
Substituting (67) into 66, we get

$$
F_{W_{k}^{(J)}}(t)=\beta_{k}^{(J)}\left[\begin{array}{c}
\gamma\left(L_{E_{k}} m_{S_{J} E_{k}}, \frac{\lambda_{S_{J} E_{k}}}{\bar{\gamma}_{S_{J}}} t\right) \\
\times \gamma\left(m_{S_{J} P}, \varphi_{J}\right)+\Delta_{k}^{(J)}(t)
\end{array}\right] .
$$

Now, it remains to compute $\mathcal{V}^{(h)}(y)$ so as to evaluate $65 \mathrm{~b}$. Using 68 , yields

$$
\mathcal{V}^{(h)}(y)=\beta_{k}^{(J)}\left(\gamma\left(m_{S_{J} P}, \varphi_{J}\right) \mathcal{T}_{1}^{(h)}+\mathcal{T}_{2}^{(h)}\right)
$$

where

$$
\begin{aligned}
& \mathcal{T}_{1}^{(h)}=\int_{0}^{\infty} t^{h} e^{-\frac{y \lambda_{S_{i} E_{k}}}{\Phi(x)} t} \gamma\left(L_{E_{k}} m_{S_{J} E_{k}}, \frac{\lambda_{S_{J} E_{k}}}{\bar{\gamma}_{S_{J}}} t\right) d t \\
& \stackrel{(a)}{=} \int_{0}^{\infty} t^{h} e^{-\frac{y \lambda_{S_{i} E_{k}}}{\Phi(x)} t} \\
& \times G_{1,2}^{1,1}\left(\begin{array}{c|c}
\frac{\lambda_{S_{J} E_{k}}}{\bar{\gamma}_{S_{J}}} t & 1 ;- \\
L_{E_{k}} m_{S_{J} E_{k}} ; 0
\end{array}\right) d t \\
& \stackrel{(b)}{=}\left(\frac{\Phi(x)}{\lambda_{S_{i} E_{k}} y}\right)^{h+1} \Theta_{1}^{(h)}(y) \text {, }
\end{aligned}
$$

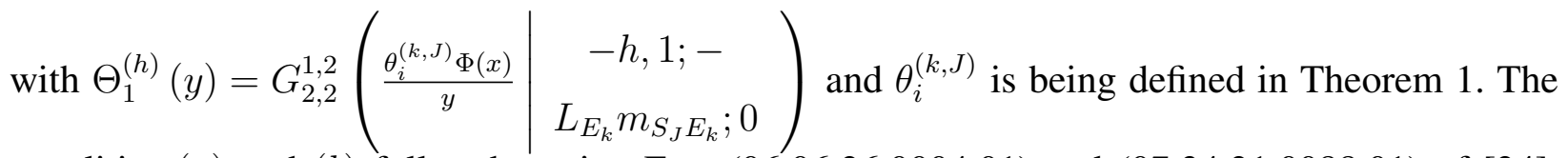
equalities $(a)$ and $(b)$ follow by using Eqs. (06.06.26.0004.01) and (07.34.21.0088.01) of [24], respectively.

On the other hand, the term $\mathcal{T}_{2}^{(h)}$ can be expressed as

$$
\begin{aligned}
\mathcal{T}_{2}^{(h)} & =\int_{0}^{\infty} t^{h} e^{-\frac{y \lambda_{S_{i} E_{k}}}{\Phi(x)} \Delta_{k}^{(J)}}(t) d t \\
& =\frac{1}{2 \pi j}\left(\frac{\Phi(x)}{\lambda_{S_{i} E_{k}} y}\right)^{h+1} \int_{\mathcal{L}_{2}} \frac{\Gamma(1+h-s) \Gamma(-s)}{\Gamma(1-s)} \\
& \times \Gamma\left(m_{S_{J} P}-s, \varphi_{J}\right) \Gamma\left(L_{E_{k}} m_{S_{J} E_{k}}+s\right)\left(\frac{\eta}{y}\right)^{-s} d s \\
& =\left(\frac{\Phi(x)}{\lambda_{S_{i} E_{k}} y}\right)^{h+1} \Theta_{2}^{(h)}(y),
\end{aligned}
$$

where $\Theta_{2}^{(h)}(y)=G_{3,2}^{1,3}\left(\begin{array}{c|c}\frac{\eta}{y} & \left(\zeta_{J}, \varphi_{J}\right),(1,0),(-h, 0) ;- \\ \left(L_{E_{k}} m_{S_{J} E_{k}}, 0\right) ;(0,0)\end{array}\right), \eta=\frac{\varsigma_{i}^{(k, J)} \Phi(x)}{\bar{\gamma}_{I}}, \zeta_{J}=1-m_{S_{J} P}$, and $\varsigma_{i}^{(k, J)}$ is being defined in Theorem 1 . 
Finally, the conditional CDF of $\gamma_{1 E}^{(i, k, J)}$ can be expressed by substituting (70) and 71) into (69) and then replacing it into 65b), yields

$$
\begin{aligned}
F_{\gamma_{1 E}^{(i, k, J)} \mid g_{S_{i} P}=x}(y) & =1-\Psi_{i}^{(k)}(y) \beta_{k}^{(J)} \\
& \times \sum_{h=0}^{L_{E_{k}} m_{S_{i} E_{k}}-1} \frac{\left(\begin{array}{c}
L_{E_{k}} m_{S_{i} E_{k}}-1 \\
h
\end{array}\right) \Phi^{h+1}(x)}{\left(\lambda_{S_{i} E_{k}} y\right)^{h+1}} \\
& \times\left[\begin{array}{c}
\gamma\left(m_{S_{J} P}, \varphi_{J}\right) \Theta_{1}^{(h)}(y) \\
+\Theta_{2}^{(h)}(y)
\end{array}\right] .
\end{aligned}
$$

Now, the remaining last previous step in this proof consists of computing $\Xi_{1}^{(i, k, J)}(x)$. Indeed, by differentiating (60) and using (1) alongside with (72), (58) can be rewritten for a positive integer $L_{R} m_{S_{i} R}$ as

$$
\begin{aligned}
\Xi_{1}^{(i, k, J)}(x) & =\frac{\Gamma\left(L_{R} m_{S_{i} R}, \frac{\xi_{S_{i} R}}{\Phi(x)}\right)}{\gamma \Gamma\left(L_{R} m_{S_{i} R}\right)} \\
& -\alpha_{i}^{(k, J)} \sum_{h=0}^{L_{E_{k}} m_{S_{i} E_{k}}-1} \frac{\Omega_{h}^{(i, k)} e^{-\frac{\xi_{S_{i} R}}{\Phi(x)}}}{(\Phi(x))^{L_{E_{k}} m_{S_{i} E_{k}}+L_{R} m_{S_{i} R}-h-2}} \\
& \times \sum_{l=0}^{L_{R} m_{S_{i} R}-1} \Upsilon_{l}^{(i)}\left[\gamma\left(m_{S_{J} P}, \varphi_{J}\right) \mathcal{U}_{1}^{(h, l)}+\mathcal{U}_{2}^{(h, l)}\right],
\end{aligned}
$$

where $\xi_{S_{i} R}, \Omega_{h}^{(i, k)}, \alpha_{i}^{(k, J)}$, and $\Upsilon_{l}^{(i)}$ are defined in 30, $32,33,, 35$, respectively, and

$$
\mathcal{U}_{a}^{(h, l)}=\int_{0}^{\infty} y^{L_{E_{k}} m_{S_{i} E_{k}}+l-h-1} e^{-\frac{\varpi_{i}^{(k)}}{\Phi(x)} y} \Theta_{a}^{(h)}(y) d y, a=\{1,2\},
$$

with $\varpi_{i}^{(k)}$ is being defined in Theorem 1 .

The two above terms can be expressed as

$$
\begin{gathered}
\mathcal{U}_{1}^{(h, l)}=\left(\frac{\Phi(x)}{\varpi_{i}^{(k)}}\right)^{l+L_{E_{k}} m_{S_{i} E_{k}}-h} \mathcal{M}_{1}^{(h, l)}\left(\varpi_{i}^{(k)} \theta_{i}^{(k, J)}\right), \\
\mathcal{U}_{2}^{(h, l)}=\frac{1}{2 \pi j} \int_{\mathcal{L}_{3}} \frac{\Gamma\left(L_{E_{k}} m_{S_{i} E_{k}}+l-h+s\right) \Gamma\left(m_{S_{J} P}-s, \varphi_{J}\right)}{\left(\frac{\Phi(x)}{\varpi_{i}^{(k)}}\right)^{-L_{E_{k}} m_{S_{i} E_{k}}-l+h} \Gamma(1-s)} \\
\times \frac{\Gamma\left(L_{E_{k}} m_{S_{J} E_{k}}+s\right) \Gamma(-s) \Gamma(1+h-s)}{\left(\frac{\varsigma_{i}^{(k, J)} \varpi_{i}^{(k)}}{\bar{\gamma}_{I}}\right)^{s}} d s, \\
=\left(\frac{\Phi(x)}{\varpi_{i}^{(k)}}\right)^{L_{E_{k}} m_{S_{i} E_{k}}+l-h} \mathcal{M}_{2}^{(h, l)}\left(\frac{\varsigma_{i}^{(k, J)} \varpi_{i}^{(k)}}{\bar{\gamma}_{I}}\right),
\end{gathered}
$$


where $\mathcal{M}_{1}^{(h, l)}(\bullet)$ and $\mathcal{M}_{2}^{(h, l)}(\bullet)$ are defined in 27, and 28, respectively. Note that 75 follows relying on [24, Eq. (07.34.21.0088.01)].

Henceforth, substituting (75) and (76) into (73), yields

$$
\begin{aligned}
\Xi_{1}^{(i, k, J)}(x) & =\frac{\Gamma\left(L_{R} m_{S_{i} R} \frac{\xi_{S_{i} R}}{\Phi(x)}\right)}{\gamma \Gamma\left(L_{R} m_{S_{i} R}\right)} \\
& -\alpha_{i}^{(k, J)} \sum_{h=0}^{L_{E_{k}} m_{S_{i} E_{k}}-1} \frac{\Omega_{h}^{(i, k)} e^{-\frac{\xi_{S_{i} R}}{\Phi(x)}}}{(\Phi(x))^{L_{E_{k}} m_{S_{i} E_{k}}+L_{R} m_{S_{i} R^{-}-h-2}}} \\
& \times \sum_{l=0}^{L_{R} m_{S_{i} R}-1} \Upsilon_{l}^{(i)}\left(\frac{\Phi(x)}{\varpi_{i}^{(k)}}\right)^{L_{E_{k}} m_{S_{i} E_{k}}+l-h} \\
& \times\left[\begin{array}{c}
\gamma\left(m_{S_{J} P}, \varphi_{J}\right) \mathcal{M}_{1}^{(h, l)}\left(\varpi_{i}^{(k)} \theta_{i}^{(k, J)}\right) \\
+\mathcal{M}_{2}^{(h, l)}\left(\frac{\varsigma_{i}^{(k, J)} \varpi_{i}^{(k)}}{\bar{\gamma}_{P}}\right)
\end{array}\right]
\end{aligned}
$$

Now, replacing (77) into (57), we obtain

$$
\begin{aligned}
S O P_{1}^{(i, k, J)} & =1-\frac{\Lambda_{1}}{\Gamma\left(L_{R} m_{S_{i} R}\right)}+\gamma \alpha_{i}^{(k, J)} \\
& \times \sum_{h=0}^{L_{E_{k}} m_{S_{2} E_{k}}-1} \Omega_{h}^{(i, k)} \sum_{l=0}^{L_{R} m_{S_{i} R^{2}-1}} \frac{\Upsilon_{l}^{(i)} \Lambda_{2}}{\left(\varpi_{i}^{(k)}\right)^{l+L_{E_{k}} m_{S_{i} E_{k}}-h}} \\
& \times\left[\begin{array}{c}
\gamma\left(m_{S_{J} P}, \varphi_{J}\right) \mathcal{M}_{1}^{(h, l)}\left(\varpi_{i}^{(k)} \theta_{i}^{(k, J)}\right) \\
+\mathcal{M}_{2}^{(h, l)}\left(\frac{\varsigma_{i}^{(k, J)} \varpi_{i}^{(k)}}{\bar{\gamma}_{I}}\right)
\end{array}\right],
\end{aligned}
$$

where

$$
\begin{aligned}
\Lambda_{1} & =\int_{0}^{\infty} f_{g_{S_{i} P}}(x) \Gamma\left(L_{R} m_{S_{i} R}, \frac{\xi_{S_{i} R}}{\Phi(x)}\right) d x \\
& \stackrel{(a)}{=} \frac{1}{\Gamma\left(m_{S_{i} P}\right)}\left[\begin{array}{c}
\gamma\left(m_{S_{i} P}, \varphi_{S_{i}}\right) \Gamma\left(L_{R} m_{S_{i} R} \frac{\xi_{S_{i} R}}{\bar{\gamma}_{S_{i}}}\right) \\
+\mathcal{M}_{3}\left(\frac{\xi_{S_{i} R}}{\lambda_{S_{i} P} \bar{\gamma}_{P}}\right)
\end{array}\right],
\end{aligned}
$$




$$
\begin{aligned}
\Lambda_{2} & =\int_{0}^{\infty} \frac{f_{g_{S_{i} P}}(x) e^{-\frac{\xi_{S_{i} R}}{\Phi(x)}}}{(\Phi(x))^{L_{R} m_{S_{i} R}-l-1}} d x \\
& =\frac{\gamma\left(m_{S_{i} P}, \varphi_{S_{i}}\right) e^{-\frac{\xi_{S_{i} R}}{\bar{\gamma}_{S_{i}}}}}{\Gamma\left(m_{S_{i} P}\right) \bar{\gamma}_{S_{i}}^{L_{S_{S}} m_{S_{i}}-l-1}}+\frac{\lambda_{S_{i} P}^{m_{S_{i} P}}}{\Gamma\left(m_{S_{i} P}\right) \bar{\gamma}_{I}^{L_{R} m_{S_{i} R}-l-1}} \\
& \times \frac{\Gamma\left(m_{S_{i} P}+L_{R} m_{S_{i} R}-l-1, \varphi_{S_{i}}+\frac{\xi_{S_{i} R}}{\bar{\gamma}_{S_{i}}}\right)}{\left(\lambda_{S_{i} P}+\frac{\xi_{S_{i} R}}{\bar{\gamma}_{P}}\right)^{m_{S_{i} P}+L_{R} m_{S_{i} R}-l-1}}
\end{aligned}
$$

with $\mathcal{M}_{3}(\bullet)$ is defined in $(31)$. Equality $(a)$ holds by replacing $\Phi(x)$ by their values and along using [24, Eqs. (06.06.26.0005.01), (07.34.21.0088.01)].

By substituting (79) and (80) into (78), (24) is attained.

\section{B. Expression of SOP at the Second Hop}

In like manner to $S O P_{1}^{(i, k)}, S O P_{2}^{(k)}$ can be expressed as

$$
S O P_{2}^{(k)}=1-\gamma \int_{0}^{\infty} f_{g_{R P}}(x) \Xi_{3}^{(k)}(x) d x,
$$

with

$$
\Xi_{3}^{(k)}(x)=\int_{0}^{\infty} f_{\gamma_{D} \mid g_{R P}=x}(\gamma+\gamma y-1) F_{\gamma_{2 E}^{(k)} \mid g_{R P}=x}(y) d y
$$

One can notice from 82 that in order to calculate $S O P_{2}^{(k)}$, it is necessary to find first the conditional CDFs of $\gamma_{D}$ and $\gamma_{2 E}^{(k)}$ for a given $g_{R P}$.

- Conditional CDFs of $\gamma_{D}$ and $\gamma_{2 E}^{(k)}$

Let's define $Y_{R D}=\sum_{t=1}^{L} g_{R D_{t}}$. In a similar manner to 60 , the conditional CDFs of $\gamma_{D}$ and $\gamma_{2 E}^{(k)}$ are given, respectively, by

$$
\begin{gathered}
F_{\gamma_{D} \mid g_{R P}=x}(z)=F_{Y_{R D}}\left(\frac{z}{\mathcal{D}(x)}\right), \\
F_{\gamma_{2 E}^{(k)} \mid g_{R P}=x}(y)=F_{\mathcal{E}_{R E_{k}}}\left(\frac{y}{\mathcal{D}(x)}\right),
\end{gathered}
$$

where $\mathcal{E}_{R E_{k}}=\sum_{u=1}^{L_{E_{k}}} g_{R E_{k}^{(u)}}, \mathcal{D}(x)=\bar{\gamma}_{R}$ for $x \leq \bar{\gamma}_{I} / \bar{\gamma}_{R}$ and $\mathcal{D}(x)=\bar{\gamma}_{I} / x$ for $x>\bar{\gamma}_{I} / \bar{\gamma}_{R}$. It follows, in a similar manner to $\mathcal{E}_{S_{i} R}$, that $\mathcal{E}_{R E_{k}}$ is also Gamma distributed with parameters $L_{E_{k}} m_{R E_{k}}$ and $\lambda_{R E_{k}}$.

- Expression of $\Xi_{3}^{(k)}(x)$ 
It is worthwhile that $Y_{R D}$ is Gamma distributed for i.i.d Nakagami- $m$ random variables with shape and scale parameters $L_{D} m_{R D}$ and $\lambda_{R D}$, respectively. That is

$$
\begin{aligned}
& \Xi_{3}^{(k)}(x) \stackrel{(a)}{=} \frac{\lambda_{R D}^{L_{D} m_{R D}} e^{-\frac{\xi_{R D}}{\mathcal{D}(x)}}}{\Gamma\left(L_{D} m_{R D}\right) \Gamma\left(L_{E_{k}} m_{R E_{k}}\right)(\mathcal{D}(x))^{L_{D} m_{R D}-1}} \\
& \times \sum_{j=0}^{L_{D} m_{R D}-1} \mathcal{B}_{j} \gamma^{j} \int_{0}^{\infty} y^{j} e^{-\frac{\lambda_{R D} \gamma}{\mathcal{D}(x)} y} \gamma\left(L_{E_{k}} m_{R E_{k}}, \frac{\lambda_{R E_{k}}}{\mathcal{D}(x)} y\right) \\
& \stackrel{(b)}{=} \frac{\lambda_{R D}^{L_{D} m_{R D}} e^{-\frac{\xi_{R D}}{\mathcal{D}(x)}}}{\gamma \Gamma\left(L_{D} m_{R D}\right) \Gamma\left(L_{E_{k}} m_{R E_{k}}\right) \mathcal{D}^{L_{D} m_{R D}-1}(x)}
\end{aligned}
$$

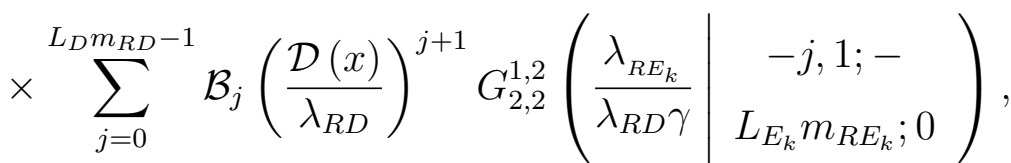

where $\mathcal{B}_{j}$ is defined in $(36)$. Note that step $(a)$ holds by substituting $(83)$ and 84 into $(82)$, while equality $(b)$ follows by using [24, Eqs. (06.06.26.0004.01), (07.34.21.0088.01)].

Substituting (85) into (81), yields

$$
\begin{aligned}
S O P_{2}^{(k)} & =1-\frac{\lambda_{R D}^{L_{D} m_{R D}}}{\Gamma\left(L_{E_{k}} m_{R E_{k}}\right) \Gamma\left(L_{D} m_{R D}\right)} \sum_{j=0}^{L_{D} m_{R D}-1} \frac{\mathcal{B}_{j}}{\lambda_{R D}^{j+1}} \\
& \times \mathcal{J}_{j} G_{2,2}^{1,2}\left(\begin{array}{c|c}
\frac{\lambda_{R E_{k}}}{\lambda_{R D} \gamma} & -j, 1 ;- \\
L_{E_{k}} m_{R E_{k}} ; 0
\end{array}\right),
\end{aligned}
$$

where

$$
\begin{gathered}
\mathcal{J}_{j}=\int_{0}^{\infty} f_{g_{R P}}(x)(\mathcal{D}(x))^{-L_{D} m_{R D}+j+1} e^{-\frac{\xi_{R D}}{\mathcal{D}(x)}} d x \\
\stackrel{(a)}{=} \frac{1}{\Gamma\left(m_{R P}\right)}\left[\begin{array}{c}
\frac{e^{-\frac{\xi_{R D}}{\bar{\gamma}_{R}}} \gamma\left(m_{R P}, \varphi_{R}\right)}{\bar{\gamma}_{R}^{L_{D} m_{R D}-j-1}} \\
+\frac{\lambda_{R P}^{m_{R P}} \Gamma\left(v_{j}, \varphi_{R}+\frac{\xi_{R D}}{\bar{\gamma}_{R}}\right)}{\bar{\gamma}_{I}^{L} D^{m} D^{-j-1}\left(\frac{\xi_{R D}}{\bar{\gamma}_{I}}+\lambda_{R P}\right)^{v_{j}}}
\end{array}\right],
\end{gathered}
$$

where $v_{j}=L_{D} m_{R D}+m_{R P}-j-1, \varphi_{R}$ is defined in Theorem 1. Here step $(a)$ is obtained by replacing $\mathcal{D}(x)$ by its values and using (1) alongside with Eqs. (3.381.1) and (3.381.3) of [21].

By considering $\frac{\bar{\gamma}_{I}}{\bar{\gamma}_{R}}=\delta$ and substituting $\sqrt{87}$ into $(86)$, one can obtain $(26)$ which concludes the proof.

\section{APPENDIX B: PROOF OF THEOREM 2}

In this section, we make use of the residues theorem in order to find the approximate expressions of Meijer-G's function given in (24). 
A. Asymptotic Expression of $S O P_{1}^{(i, k, J)}$

1) Case 1: Presence of Jammer: The Meijer-G's functions $\mathcal{M}_{1}^{(h, l)}(z)$ and $\mathcal{M}_{2}^{(h, l)}(z)$ given in (24) can be expressed in terms of complex integral as

$$
\begin{aligned}
\mathcal{M}_{1}^{(h, l)}(z) & =\frac{1}{2 \pi j} \int_{\mathcal{L}_{3}} \frac{\Gamma\left(L_{E_{k}} m_{S_{J} E_{k}}+s\right) \Gamma(1+h-s)}{\Gamma(1-s)} \\
& \times \Gamma\left(L_{E_{k}} m_{S_{i} E_{k}}+l-h+s\right) \Gamma(-s) z^{-s} d s,
\end{aligned}
$$

and

$$
\begin{aligned}
\mathcal{M}_{2}^{(h, l)}(z) & =\frac{1}{2 \pi j} \int_{\mathcal{L}_{3}} \frac{\Gamma\left(L_{E_{k}} m_{S_{J} E_{k}}+s\right) \Gamma(1+h-s)}{\Gamma(1-s)} \\
& \times \Gamma\left(L_{E_{k}} m_{S_{i} E_{k}}+l-h+s\right) \Gamma(-s) \\
& \times \Gamma\left(m_{S_{J} P}-s, \varphi_{J}\right) z^{-s} d s .
\end{aligned}
$$

It is noteworthy that the conditions of [22, Theorem 1.5] are satisfied. That is, the two above functions can be written as an infinite sum of the poles belonging to the left half plan of $\mathcal{L}_{3}$. Furthermore, as the upper incomplete gamma function in $(89)$ is always finite for $\varphi_{J} \neq 0$, it follows that the integrand functions of the two above equations have the same poles. Additionally, it is clearly seen that the order of the left poles depends on the values of $L_{E_{k}} m_{S_{J} E_{k}}, L_{E_{k}} m_{S_{i} E_{k}}$, $h$, and $l$. Owing to this fact, three cases can be distinguished:

- $-L_{E_{k}} m_{S_{J} E_{k}}<-L_{E_{k}} m_{S_{i} E_{k}}-l+h$ : In this case, the two integrand functions given in 88 and (89) admit $-\chi_{h, l, r}$ with $\chi_{h, l, r}=L_{E_{k}} m_{S_{i} E_{k}}+l-h+r$ and $0 \leq r \leq L_{E_{k}} m_{S_{J} E_{k}}-$ $L_{E_{k}} m_{S_{i} E_{k}}-l+h-1$ as simple poles and $-\varrho_{r}$ with $\varrho_{r}=L_{E_{k}} m_{S_{J} E_{k}}+r$ and $r$ natural number as poles of second-order.

- $-L_{E_{k}} m_{S_{J} E_{k}}>-L_{E_{k}} m_{S_{i} E_{k}}-l+h:$ Under this condition, the aforementioned integrands have $-\varrho_{r}$ with $0 \leq r \leq L_{E_{k}} m_{S_{i} E_{k}}-L_{E_{k}} m_{S_{J} E_{k}}+l-h-1$ as simple poles and $-\chi_{h, l, r}$ where $r \in \mathbb{N}$ as poles of second-order.

- $-L_{E_{k}} m_{S_{J} E_{k}}=-L_{E_{k}} m_{S_{i} E_{k}}-l+h$ : Under this assumption, the two integrands admit only poles of second-order at $-\varrho_{r}, r \in \mathbb{N}$.

a. $-L_{E_{k}} m_{S_{J} E_{k}}<-L_{E_{k}} m_{S_{i} E_{k}}-\mathbf{l}+\mathbf{h}$

Relied on [22, Theorem 1.5], $\mathcal{M}_{1}^{(h, l)}(z)$ can be rewritten as series of residues at the aforementioned poles 


$$
\begin{aligned}
\mathcal{M}_{1}^{(h, l)}(z) & =\sum_{r=0}^{\varrho_{r}-\chi_{h, l, r}-1} \lim _{s \rightarrow-\chi_{h, l, r}} Q_{1}(s, z) \\
& +\sum_{r=0}^{\infty} \lim _{s \rightarrow-\varrho_{r}} \frac{\partial Q_{2}(s, z)}{\partial s}
\end{aligned}
$$

where

$$
\begin{aligned}
Q_{1}(s, z) & =\left(\chi_{h, l, r}+s\right) \Gamma\left(\chi_{h, l, r}-r+s\right) \\
& \times \frac{\Gamma\left(L_{E_{k}} m_{S_{J} E_{k}}+s\right) \Gamma(-s) \Gamma(1+h-s)}{\Gamma(1-s)} z^{-s},
\end{aligned}
$$

and

$$
\begin{aligned}
Q_{2}(s, z) & =\left(\varrho_{r}+s\right)^{2} \Gamma\left(L_{E_{k}} m_{S_{i} E_{k}}+l-h+s\right) \\
& \times \frac{\Gamma\left(L_{E_{k}} m_{S_{J} E_{k}}+s\right) \Gamma(-s) \Gamma(1+h-s)}{\Gamma(1-s)} z^{-s} .
\end{aligned}
$$

Obviously, the limit of $Q_{1}(s, z)$ can be expressed as

$$
\begin{aligned}
\lim _{s \rightarrow-\chi_{h, l, r}} Q_{1}(s, z) & =\frac{(-1)^{r} \Gamma\left(L_{E_{k}} m_{S_{i} E_{k}}+l+r+1\right)}{r ! \chi_{h, l, r}} \\
& \times \Gamma\left(L_{E_{k}} m_{S_{J} E_{k}}-\chi_{h, l, r}\right) z^{\chi_{h, l, r}} .
\end{aligned}
$$

On the other hand, using [24, Eqs. (06.14.06.0026.01) and (06.14.16.0003.01)] the partial derivative of $Q_{2}(s, z)$ is given by

$$
\begin{aligned}
\frac{\partial Q_{2}(s, z)}{\partial s} & =\frac{\left(s+\varrho_{r}\right)^{2} \Gamma\left(\chi_{h, l, r}-r+s\right) \Gamma(-s)}{\Gamma(1-s) z^{s}} \\
& \times \Gamma\left(L_{E_{k}} m_{S_{J} E_{k}}+s\right) \Gamma(1+h-s) \mathcal{G}^{(h, l, r)}(z, s),
\end{aligned}
$$

where $\mathcal{G}^{(h, l, r)}(z, s)=-\log z+\psi(r+1)+\psi\left(\varrho_{r}-\chi_{h, l, r}+r+1\right)-\frac{1}{s}-\psi(1+h-s)$.

Replacing (94) and (93) into (90), yields

$$
\begin{aligned}
\mathcal{M}_{1}^{(h, l)}(z) & =\sum_{r=0}^{\varrho_{r}-\chi_{h, l, r}-1} \frac{(-1)^{r} \Gamma\left(L_{E_{k}} m_{S_{J} E_{k}}-\chi_{h, l, r}\right)}{r ! \chi_{h, l, r}} \\
& \times \Gamma\left(L_{E_{k}} m_{S_{i} E_{k}}+l+r+1\right) z^{\chi_{h, l, r}} \\
& +\sum_{r=0}^{\infty} \frac{(-1)^{\varrho_{r}-\chi_{h, l, r}} \Gamma\left(h+\varrho_{r}+1\right)}{\left(-L_{E_{k}} m_{S_{i} E_{k}}+\varrho_{r}-l+h\right) ! k ! \varrho_{r}} \\
& \times z^{\varrho_{r}} \mathcal{G}^{(h, l, r)}\left(z,-\varrho_{r}\right) .
\end{aligned}
$$


In similar manner, $\mathcal{M}_{2}^{(h, l)}(z)$ can be expressed as

$$
\begin{aligned}
\mathcal{M}_{2}^{(h, l)}(z) & =\sum_{k=0}^{\varrho_{r}-\chi_{h, l, r}-1} \frac{(-1)^{r} \Gamma\left(L_{E_{k}} m_{S_{i} E_{k}}+l+r+1\right)}{r ! \chi_{h, l, r} z^{-\chi_{h, l, r}}} \\
& \times \Gamma\left(m_{S_{J} P}+\chi_{h, l, r}, \varphi_{J}\right) \Gamma\left(L_{E_{k}} m_{S_{J} E_{k}}-\chi_{h, l, r}\right) \\
& +\sum_{r=0}^{\infty} \frac{(-1)^{\varrho_{r}-\chi_{h, l, r}} \Gamma\left(1+h+\varrho_{r}\right)}{\left(-L_{E_{k}} m_{S_{i} E_{k}}+\varrho_{r}-l+h\right) ! r ! \varrho_{r}} z^{\varrho_{r}} \\
& \times\left\{\begin{array}{c}
{\left[\mathcal{G}^{(h, l, r)}\left(z,-\varrho_{r}\right)-\log \left(\varphi_{J}\right)\right]} \\
\times \Gamma\left(m_{S_{J} P}+\varrho_{r}, \varphi_{J}\right)-\mathcal{V}^{(r)}\left(\varrho_{r}\right)
\end{array}\right\}
\end{aligned}
$$

where $\mathcal{V}^{(r)}\left(\varrho_{r}\right)=G_{2,3}^{3,0}\left(\varphi_{J} \mid \begin{array}{c}-; 1,1 \\ 0,0, m_{S_{J} P}+\varrho_{r} ;-\end{array}\right)$.

Interestingly, one can notice that $\varrho_{r}>\chi_{h, l, r}$ for $L_{E_{k}} m_{S_{J} E_{k}}>L_{E_{k}} m_{S_{i} E_{k}}+l-h$. Consequently, the second summation in the two above expressions can be ignored as $z$ approaches 0 , i.e.,

$$
\begin{aligned}
\mathcal{M}_{1}^{(h, l)}(z) & \sim \frac{\Gamma\left(L_{E_{k}}\left(m_{S_{J} E_{k}}-m_{S_{i} E_{k}}\right)-l+h\right)}{\left(L_{E_{k}} m_{S_{i} E_{k}}+l-h\right)}, \\
& \times \Gamma\left(L_{E_{k}} m_{S_{i} E_{k}}+l+1\right) z^{L_{E_{k}} m_{S_{i} E_{k}}+l-h}
\end{aligned}
$$

and

$$
\begin{aligned}
\mathcal{M}_{2}^{(h, l)}(z) & \sim \frac{\Gamma\left(L_{E_{k}} m_{S_{i} E_{k}}+l+1\right) z^{L_{E_{k}} m_{S_{i} E_{k}}+l-h}}{\left(L_{E_{k}} m_{S_{i} E_{k}}+l-h\right)} \\
& \times \Gamma\left(L_{E_{k}}\left(m_{S_{J} E_{k}}-m_{S_{i} E_{k}}\right)-l+h\right) \\
& \times \Gamma\left(m_{S_{J} P}+L_{E_{k}} m_{S_{i} E_{k}}+l-h, \varphi_{J}\right) .
\end{aligned}
$$

b. $-L_{E_{k}} m_{S_{J} E_{k}}>-L_{E_{k}} m_{S_{i} E_{k}}-\mathbf{l}+\mathbf{h}$

Analogously to the previous case, the integrals 88 and 89 can be computed relied on [22, 
Theorem 1.5] and [24, Eq. (06.14.16.0003.01)], respectively, as

$$
\begin{aligned}
\mathcal{M}_{1}^{(h, l)}(z) & =\sum_{r=0}^{\chi_{h, l, r}-\varrho_{r}-1} \frac{\Gamma\left(L_{E_{k}} m_{S_{i} E_{k}}+l-h-\varrho_{r}\right) \Gamma\left(1+h+\varrho_{r}\right)}{r ! \varrho_{r}(-1)^{-r} z^{-\varrho_{r}}} \\
& +\sum_{r=0}^{\infty} \frac{(-1)^{\chi_{h, l, r}-\varrho_{r}} \Gamma\left(1+h+\chi_{h, l, r}\right)}{\left(\chi_{h, l, r}-L_{E_{k}} m_{S_{J} E_{k}}\right) ! r ! \chi_{h, l, r} z^{-\chi_{h, l, r}}} \\
& \times\left\{\begin{array}{c}
\psi(r+1)+\psi\left(-L_{E_{k}} m_{S_{J} E_{k}}+\chi_{h, l, r}+1\right) \\
-\psi\left(1+L_{E_{k}} m_{S_{i} E_{k}}+l+r\right)+\frac{1}{\chi_{h, l, r}}-\log z
\end{array}\right\},
\end{aligned}
$$

and

$$
\begin{aligned}
\mathcal{M}_{2}^{(h, l)}(z) & =\sum_{r=0}^{\chi_{h, l, r}-\varrho_{r}-1} \frac{(-1)^{r} \Gamma\left(L_{E_{k}} m_{S_{i} E_{k}}+l-h-\varrho_{r}\right)}{r ! \varrho_{r}} \\
& \times \Gamma\left(1+h+\varrho_{r}\right) z^{\varrho_{r}} \Gamma\left(m_{S_{J} P}+\varrho_{r}, \varphi_{J}\right) \\
& +\sum_{r=0}^{\infty} \frac{(-1)^{\chi_{h, l, r}-\varrho_{r}} \Gamma\left(1+h+\chi_{h, l, r}\right)}{\left(\chi_{h, l, r}-\varrho_{r}\right) ! r ! \chi_{h, l, r} z^{-\chi_{h, l, r}}} \\
& \times\left[\mathcal{Z}-\mathcal{V}^{(r)}\left(\chi_{h, l, r}\right)\right]
\end{aligned}
$$

where

$$
\mathcal{Z}=\Gamma\left(m_{S_{J} P}+\chi_{h, l, r}, \varphi_{J}\right)\left[\begin{array}{c}
-\log z+\psi(r+1) \\
+\psi\left(-L_{E_{k}} m_{S_{J} E_{k}}+\chi_{h, l, r}+1\right) \\
-\psi\left(L_{E_{k}} m_{S_{i} E_{k}}+l-h+r\right) \\
-\psi\left(L_{E_{k}} m_{S_{i} E_{k}}+l+r+1\right) \\
+\psi\left(1+\chi_{h, l, r}\right)-\log \left(\varphi_{J}\right)
\end{array}\right]
$$

One can notice that as $\varrho_{r}<\chi_{h, l, r}, \mathcal{M}_{1}^{(h, l)}(z)$ and $\mathcal{M}_{2}^{(h, l)}(z)$ can be approximated when $z$ tends to 0

$$
\begin{aligned}
\mathcal{M}_{1}^{(h, l)}(z) & \sim \frac{\Gamma\left(L_{E_{k}} m_{S_{i} E_{k}}-L_{E_{k}} m_{S_{J} E_{k}}+l-h\right)}{L_{E_{k}} m_{S_{J} E_{k}}} \\
& \times \Gamma\left(L_{E_{k}} m_{S_{J} E_{k}}+h+1\right) z^{L_{E_{k}} m_{S_{J} E_{k}}}
\end{aligned}
$$

and

$$
\begin{aligned}
\mathcal{M}_{2}^{(h, l)}(z) & \sim \frac{\Gamma\left(L_{E_{k}} m_{S_{J} E_{k}}+h+1\right) \Gamma\left(m_{S_{J} P}+L_{E_{k}} m_{S_{J} E_{k}}, \varphi_{J}\right)}{L_{E_{k}} m_{S_{J} E_{k}}} \\
& \times \Gamma\left(L_{E_{k}} m_{S_{i} E_{k}}-L_{E_{k}} m_{S_{J} E_{k}}+l-h\right) z^{L_{E_{k}} m_{S_{J} E_{k}}}
\end{aligned}
$$


c. $-L_{E_{k}} m_{S_{J} E_{k}}=-L_{E_{k}} m_{S_{i} E_{k}}-l+h$

For this case, the two complex integrals given in $(88)$ and $(89)$ can be expressed by performing some algebraic operations as

$$
\begin{aligned}
\mathcal{M}_{1}^{(h, l)}(z) & =\sum_{r=0}^{\infty} \frac{(-1)^{\varrho_{r}-\chi_{h, l, r}} \Gamma\left(1+h+\varrho_{r}\right)}{\left(-L_{E_{k}} m_{S_{i} E_{k}}+\varrho_{r}-l+h\right) ! k ! \varrho_{r}} \\
& \times z^{\varrho_{r}} \mathcal{G}^{(h, l, r)}\left(z,-\varrho_{r}\right) .
\end{aligned}
$$

and

$$
\begin{aligned}
\mathcal{M}_{2}^{(h, l)}(z) & =\sum_{r=0}^{\infty} \frac{(-1)^{\varrho_{r}-\chi_{h, l, r}} \Gamma\left(1+h+\varrho_{r}\right)}{\left(\varrho_{r}-L_{E_{k}} m_{S_{i} E_{k}}-l+h\right) ! r ! \varrho_{r}} z^{\varrho_{r}} \\
& \times\left\{\begin{array}{c}
{\left[\mathcal{G}^{(h, l, r)}(z)-\log \left(\varphi_{J}\right)\right]} \\
\times \Gamma\left(m_{S_{J} P}+\varrho_{r}, \varphi_{J}\right)-\mathcal{V}^{(r)}\left(\varrho_{r}\right)
\end{array}\right\} .
\end{aligned}
$$

Again, $\mathcal{M}_{1}^{(h, l)}(z)$ and $\mathcal{M}_{2}^{(h, l)}(z)$ can be approximated as $z$ approaches 0 by

$$
\begin{aligned}
\mathcal{M}_{1}^{(h, l)}(z) & \sim \frac{(-1)^{L_{E_{k}} m_{S_{J} E_{k}}-L_{R} m_{S_{i} R}+1} z^{L_{E_{k}} m_{S_{J} E_{k}} \log z}}{L_{E_{k}} m_{S_{J} E_{k}}} \\
& \times \Gamma\left(L_{E_{k}} m_{S_{i} E_{k}}+L_{E_{k}} m_{S_{J} E_{k}}\right)
\end{aligned}
$$

and

$$
\begin{aligned}
\mathcal{M}_{2}^{(h, l)}(z) & \sim \frac{(-1)^{L_{E_{k}} m_{S_{J} E_{k}}-L_{R} m_{S_{i} R}+1} \Gamma\left(L_{E_{k}}\left(m_{S_{i} E_{k}}+m_{S_{J} E_{k}}\right)\right)}{L_{E_{k}} m_{S_{J} E_{k}}} \\
& \times \Gamma\left(m_{S_{J} P}+L_{E_{k}} m_{S_{J} E_{k}}, \varphi_{J}\right) z^{L_{E_{k}} m_{S_{J} E_{k}}} \log (z) .
\end{aligned}
$$

Finally, the Meijer's G-function $\mathcal{M}_{3}(z)$ defined in (31) can be written in terms of complex integral as

$$
\mathcal{M}_{3}(z)=\frac{1}{2 \pi j} \int_{\mathcal{L}_{4}} \frac{\Gamma\left(L_{R} m_{S_{i} R}+s\right) \Gamma\left(m_{S_{i} P}-s, \varphi_{S_{i}}\right)}{s} z^{-s} d s .
$$

It is worth mentioning that the conditions of [22] are applied also here. Thus, the above integrand function can be written as an infinite sum of the left poles in $\mathcal{L}_{4}$. Besides, that integrand admits only poles of the first order at 0 and $-L_{R} m_{S_{i} R}-r, r \in \mathbb{N}$. That is

$$
\begin{aligned}
\mathcal{M}_{3}(z) & =\Gamma\left(L_{R} m_{S_{i} R}\right) \Gamma\left(m_{S_{i} P}, \varphi_{S_{i}}\right) \\
& +\sum_{r=0}^{\infty} \frac{(-1)^{r+1} \Gamma\left(m_{S_{i} P}+L_{R} m_{S_{i} R}+r, \varphi_{S_{i}}\right)}{r !\left(L_{R} m_{S_{i} R}+r\right) z^{-L_{R} m_{S_{i} R^{-r}}}} \\
& \stackrel{(a)}{\sim} \Gamma\left(L_{R} m_{S_{i} R}\right) \Gamma\left(m_{S_{i} P}, \varphi_{S_{i}}\right)-\frac{\Gamma\left(m_{S_{i} P}+L_{R} m_{S_{i} R}, \varphi_{S_{i}}\right)}{L_{R} m_{S_{i} R} z^{-L_{R} m_{S_{i} R}}}
\end{aligned}
$$


with step $(a)$ follows by considering only the first term of the infinite summation when $z$ tends to 0 .

Finally, armed by [21, Eq. (8.354.2)] the upper incomplete Gamma given in (24) can be approximated for small values of $z$ as

$$
\Gamma\left(L_{R} m_{S_{i} R}, \frac{\sigma_{i} \xi_{S_{i} R}}{\bar{\gamma}_{I}}\right) \sim \Gamma\left(L_{R} m_{S_{i} R}\right)-\frac{1}{L_{R} m_{S_{i} R}}\left(\frac{\sigma_{i} \xi_{S_{i} R}}{\bar{\gamma}_{I}}\right)^{L_{R} m_{S_{i} R}}
$$

Interestingly, the $S O P_{1}^{(i, k, J)}$ can finally be approximated in high SNR regime (i.e., $\bar{\gamma}_{I} \rightarrow$ $\infty)$ by considering three cases, namely $L_{R} m_{S_{i} R}<L_{E_{k}} m_{S_{J} E_{k}}, L_{R} m_{S_{i} R}>L_{E_{k}} m_{S_{J} E_{k}}$, and $L_{R} m_{S_{i} R}=L_{E_{k}} m_{S_{J} E_{k}}$.

- $L_{R} m_{S_{i} R}<L_{E_{k}} m_{S_{J} E_{k}}$

Substituting (97), (98), (109), and (110) into (24), and by considering $h=L_{E_{k}} m_{S_{i} E_{k}}-1$, $S O P_{1}^{(i, k, J)}$ can be approximated as

$$
S O P_{1}^{(i, k, J)} \sim \frac{\mathcal{C}_{1}^{(i, k, J)}}{\bar{\gamma}_{I}^{L_{R} m_{S_{i} R}}}
$$

where $\mathcal{C}_{1}^{(i, k, J)}$ is given in 53 .

- $L_{R} m_{S_{i} R}>L_{E_{k}} m_{S_{J} E_{k}}$

Incorporating (102), (103), (109), and (110) into (24), and by considering $l=L_{R} m_{S_{i} R}-1$, $S O P_{1}^{(i, k, J)}$ can be approximated as

$$
S O P_{1}^{(i, k, J)} \sim \frac{\mathcal{C}_{2}^{(i, k, J)}}{\bar{\gamma}_{I}^{L_{E_{k}} m_{S_{J} E_{k}}}}
$$

where $\mathcal{C}_{2}^{(i, k, J)}$ is given in 54$)$.

- $L_{R} m_{S_{i} R}=L_{E_{k}} m_{S_{J} E_{k}}$

Replacing (102), (103), (106), (107), (109), and (110) into (24), and by considering $l=$ $L_{R} m_{S_{i} R}-1, S O P_{1}^{(i, k, J)}$ can be approximated as

$$
S O P_{1}^{(i, k, J)} \sim \mathcal{C}_{3}^{(i, k, J)} \frac{\log \left(\bar{\gamma}_{I}\right)}{\bar{\gamma}_{I}^{L_{E_{k}} m_{S_{J} E_{k}}}}
$$

where $\mathcal{C}_{3}^{(i, k, J)}$ is given in 55 . 
2) Case 2: Absence of a Friendly Jammer: In order to derive the asymptotic expression of $S O P_{1}^{(i, k)}$ given in 25 , we need to approximate the upper incomplete Gamma function. One can ascertain by applying the Maclaurin series that

$$
\Gamma(a, b+c z) \sim \Gamma(a, b)-c z b^{a-1} e^{-b},
$$

as $z$ tends to 0 . By considering only the two cases i.e., $l=L_{R} m_{S_{i} R}-1$ and $l=L_{R} m_{S_{i} R}-2$ and performing some algebraic manipulations, one can obtain

$$
S O P_{1}^{(i, k)} \sim 1-\mathcal{A}_{S_{i} E_{k}, S_{i} R, R}-\frac{\mathcal{A}_{S_{i} E_{k}, S_{i} R, S_{i}, R}}{\bar{\gamma}_{I}},
$$

where $\mathcal{A}_{\bullet, \bullet, \bullet}$ and $\mathcal{A}_{\bullet, \bullet, \bullet, \bullet}$ are defined in (51) and (52), respectively.

\section{B. Asymptotic Expression of $\mathrm{SOP}_{2}^{(k)}$}

As $S O P_{1}^{(i, k)}$ and $S O P_{2}^{(k)}$ given in 25 and 26, respectively have the same shape, one can see that

$$
S O P_{2}^{(k)} \sim 1-\mathcal{A}_{R E_{k}, R D}-\frac{\mathcal{A}_{R E_{k}, R D, R}}{\bar{\gamma}_{I}},
$$

Finally, replacing (111), (112), (113), and (116) into (22), one can get the expressions (37)(40), respectively. Furthermore, substituting (115) and (116) into (23), (50) is attained which concludes the proof of Theorem 2 .

\section{REFERENCES}

[1] J. Mitola, "Cognitive radio: An integrated agent architecture for software-defined radio," Doctor of Technology, Royal Inst. Technol. (KTH), Stockholm, Sweden, 2000.

[2] H. Lei, C. Gao, I. S. Ansari, Y. Guo, Y. Zou, G. Pan, and K. A. Qaraqe, "Secrecy outage performance of transmit antenna selection for MIMO underlay cognitive radio systems over Nakagami-m channels," IEEE Transactions on Vehicular Technology, vol. 66, no. 3, pp. 2237-2250, 2016.

[3] H. Tran, G. Kaddoum, F. Gagnon, and L. Sibomana, "Cognitive radio network with secrecy and interference constraints," Physical Communication, vol. 22, pp. 32-41, 2017.

[4] M. Elkashlan, L. Wang, T. Q. Duong, G. K. Karagiannidis, and A. Nallanathan, "On the security of cognitive radio networks," IEEE Transactions on Vehicular Technology, vol. 64, no. 8, pp. 3790-3795, 2014.

[5] N.-P. Nguyen, T. L. Thanh, T. Q. Duong, and A. Nallanathan, "Secure communications in cognitive underlay networks over Nakagami-m channel," Physical Communication, vol. 25, pp. 610-618, 2017. 
[6] H. Lei, H. Zhang, I. S. Ansari, Z. Ren, G. Pan, K. A. Qaraqe, and M.-S. Alouini, "On secrecy outage of relay selection in underlay cognitive radio networks over Nakagami-m fading channels," IEEE Transactions on Cognitive Communications and Networking, vol. 3, no. 4, pp. 614-627, 2017.

[7] K. Ho-Van and T. Do-Dac, "Analysis of security performance of relay selection in underlay cognitive networks," IET Communications, vol. 12, no. 1, pp. 102-108, 2017.

[8] J. Ding, Q. Yang, and J. Yang, "Secrecy outage probability of minimum relay selection in multiple eavesdroppers DF cognitive radio networks," in 2016 IEEE 83rd Vehicular Technology Conference (VTC Spring), pp. 1-5, IEEE, 2016.

[9] H. Sakran, M. Shokair, O. Nasr, S. El-Rabaie, and A. A. El-Azm, "Proposed relay selection scheme for physical layer security in cognitive radio networks," IET Communications, vol. 6, no. 16, pp. 2676-2687, 2012.

[10] P. Yan, Y. Zou, and J. Zhu, "Energy-aware multiuser scheduling for physical-layer security in energy-harvesting underlay cognitive radio Systems", IEEE Trans. on Vehicular Technology, vol. 67, no. 3, pp. 2084-2096, March 2018.

[11] M. Hanif, H. Yang, and M.-S. Alouini, "Transmit antenna selection for power adaptive underlay cognitive radio with instantaneous interference constraint", IEEE Trans. on Commun., vol. 65, no. 6, pp. 2357-2367, June 2017.

[12] H. Lei, M. Xu, I. Ansari, G. Pan, K. Qaraqe, and M.-S. Alouini, "On secure underlay MIMO cognitive radio networks with energy harvesting and transmit antenna selection", IEEE Trans. Green Commun. Netw., vol. 1, no. 2, pp. 192-203, 2017.

[13] M. Bouabdellah, F. El Bouanani, P. C. Sofotasios, D. B. da Costa, K. Mezher, H. Ben-azza, S. Muhaidat, G. K. Karagiannidis, "Physical layer security for dual-hop SWIPT-enabled CR networks", in Proceedings of the International Symposium on Wireless Communication Systems (ISWCS), Aug. 2019.

[14] M. Bouabdellah, F. El Bouanani, P. C. Sofotasios, D. B. da Costa, S. Muhaidat, K. Mezher, H. Ben-azza, and G. K. Karagiannidis, "Dual-hop cooperative energy harvesting cognitive radio networks with spectrum sharing and security constraints," IEEE Access, Nov. 2019, DOI: 10.1109/ACCESS.2019.2955205.

[15] Z. Xiang, W. Yang, G. Pan, Y. Cai and Y. Song, "Physical layer security in cognitive radio inspired NOMA network", IEEE Journal of Selected Topics in Signal Processing, vol. 13, no. 3, pp. 700-714, 2019.

[16] Y. Song, W. Yang, Z. Xiang, B. Wang and Y. Cai, "Secure transmission in mmWave NOMA networks with cognitive power allocation", IEEE Access, vol. 7, pp. 76104-76119, 2019.

[17] Y. Zou, "Physical-layer security for spectrum sharing systems," IEEE Transactions on Wireless Communications, vol. 16, no. 2, pp. 1319-1329, 2016.

[18] Y. Liu, L. Wang, T. T. Duy, M. Elkashlan, and T. Q. Duong, "Relay selection for security enhancement in cognitive relay networks," IEEE wireless communications letters, vol. 4, no. 1, pp. 46-49, 2014.

[19] M. Bouabdellah, F. El Bouanani, P. C. Sofotasios, D. B. da Costa, H. Ben-Azza, K. Mezher, S. Muhaidat, "Intercept probability of underlay uplink CRNs with multi-eavesdroppers", in Proceedings of the IEEE International Symposium on Personal, Indoor and Mobile Radio Communications (PIMRC), Sep. 2019.

[20] C. Zhong, T. Ratnarajah and K. Wong, "Outage analysis of decode-and-forward cognitive dual-hop systems With the interference constraint in Nakagami-m fading channels", IEEE Transactions on Vehicular Technology, vol. 60, no. 6, pp. 2875-2879, 2011.

[21] I. S. Gradshteyn and I. M. Ryzhik, Table of Integrals, Series, and Products: Seventh Edition. Burlington, MA: Elsevier, 2007.

[22] A. A. Kilbas, H-transforms: Theory and Applications. CRC Press, 2004.

[23] M. Bouabdellah, F. E. Bouanani, and H. Ben-Azza, "Secrecy outage performance for dual-hop underlay cognitive radio system over Nakagamim fading," in Proceedings of International Conference on Smart Digital Environment (ICSDE'18), Oct. 2018. 
[24] I. W. Research, Mathematica Edition: version 11.3. Champaign, Illinois: Wolfram Research, Inc., 2018. 